This is the Accepted Version of: Guo, Xiaojun; Xu, Yong; Ogier, Simon; [et al.]. «Current Status and Opportunities of Organic Thin-Film Transistor Technologies». IEEE Transactions on electron devices, vol.64, no 5 (2017). DOI 10.1109/ TED.2017.2677086. All rights reserved

\title{
Current Status and Opportunities of Organic Thin-Film Transistor Technologies
}

\author{
Xiaojun Guo, Member, IEEE, Yong Xu, Member, IEEE, Simon Ogier, Tse Nga Ng, Mario Caironi, \\ Andrea Perinot, Ling Li, Jiaqing Zhao, Wei Tang, Radu A. Sporea, Member, IEEE, Ahmed Nejim, Jordi \\ Carrabina, Paul Cain and Feng Yan
}

\begin{abstract}
Attributed to its advantages of super mechanical flexibility, very low temperature processing, and compatibility with low cost and high throughput manufacturing, organic thin film transistor (OTFT) technology is able to bring electrical, mechanical and industrial benefits to a wide range of new applications by activating non-flat surfaces with flexible displays, sensors and other electronic functions. Despite both strong application demand and these significant technological advances, there is still a gap to be filled for OTFT technology to be widely commercially adopted. This paper provides a comprehensive review of the current status of OTFT technologies ranging from material, device, process, and integration, to design and system applications, and clarifies the real challenges behind to be addressed.
\end{abstract}

Index Terms - flexible electronics, printed electronics, organic thin film transistor (OTFT), displays, sensors, hybrid integration

Manuscript received xxx, 2016. This work was supported by National Key R\&D Program of "Strategic Advanced Electronic Materials" under Grant No 2016YFB0401100 and the NSFC of China (Grant No. 61274083, 61334008).

Xiaojun Guo, Jiaqing Zhao and Wei Tang are with the National Engineering Laboratory of TFT-LCD Materials and Technologies, Department of Electronic Engineering, Shanghai Jiao Tong University, Shanghai 200240, China (e-mail: x.guo@sjtu.edu.cn).

Yong Xu is with Dongguk University, Department of Energy and Materials Engineering, 26 Pil-dong, 3-ga, Jung-gu, Seoul 100-715, Korea (e-mail: xu.yong@dongguk.edu)

Simon Ogier is with NeuDrive Ltd, BioHub, Alderley Park, Macclesfield SK10 4TG, UK, (e-mail: simon.ogier@neudrive.com).

Tse Nga Ng is with University of California San Diego, Department of Electrical and Computer Engineering, 9500 Gilman Dr., La Jolla, CA 92093, USA (email: tnn046@eng.ucsd.edu).

Mario Caironi and Andrea Perinot are with Center for Nano Science and Technology@PoliMi, Istituto Italiano di Technologia, via Giovanni Pascoli 70/3, Milano, Italy (email: mario.caironi@iit.it; andrea.perinot@iit.it).

Ling Li is with Institute of Microelectronics, Chinese Academy of Sciences, Beijing 100029, China (email: lingli@ime.ac.cn).

Radu A. Sporea is with the Advanced Technology Institute, University of Surrey, Guildford GU2 7XH, U.K. (e-mail: r.a.sporea@surrey.ac.uk).

Ahmed Nejim is with Silvaco Europe Ltd, Cambridgeshire PE27 5JL, UK (email: ahmed.nejim@silvaco.com).

Jordi Carrabina is with Autonomous University of Barcelona, 08192 Bellaterra (Spain). (email: jordi.carrabina@uab.cat).

Paul Cain is with FlexEnable Ltd., Milton Road, Cambridge, UK CB4 0FX (email: paul.cain@flexenable.com).

Feng Yan is with the Department of Applied Physics and Materials Research Center, The Hong Kong Polytechnic University, Hong Kong (e-mail: feng.yan@polyu.edu.hk).

\section{INTRODUCTION}

THE thin film transistor (TFT) is a key element for realizing functional large area electronics systems including displays and sensors, by providing capabilities of matrix addressing, current driving and signal processing $[1,2]$. For many years, large area manufacturing of hydrogenated amorphous silicon (a-Si) TFT has been well established in the industry for flat-panel liquid crystal displays (LCDs) and imagers [3]. To overcome the poor mobility and bias stress instabilities of a-Si TFTs, low temperature polycrystalline (LTPS) TFTs with much higher mobility and very stable electrical properties were developed, and have been successfully applied to commercial high-end LCD displays and organic light emitting diode (OLED) displays. However, the LTPS TFT requires additional high cost laser crystallization and doping processes, and suffers poor spatial uniformity of the electrical properties [4]. Manufacturing of LTPS TFTs is difficult to be scaled up to large area (e. g. > Gen 6), which limits its low cost and large area applications. Since 2004, amorphous metal oxide (AOS) semiconductor based TFTs have attracted wide attention for their advantages of high mobility, steep subthreshold and ultra-low leakage current, with low cost and large area manufacturability attributes similar to a-Si TFT $[5,6]$. With these attractive features, the AOS TFT has been quickly adopted to display manufacturing for commercial high resolution low power LCD panels and large size OLED panels. Moreover, with its low temperature processing, the AOS TFT is also regarded a very promising solution for constructing high performance large area flexible electronics $[7,8]$.

Compared to inorganic counterparts, organic TFTs hold several unique advantages for low cost and ultra-flexible electronics applications. Firstly, the stack of organic semiconductor (OSC) and polymer gate dielectric with low temperature and fast annealing processes is compatible with low cost high throughput printing-based manufacturing [9], and also provides excellent intrinsic mechanical flexibility $[10,11]$ or even stretchability $[12,13]$ for truly flexible and stretchable electronics. Secondly, OSCs have great potential for continuous performance improvement and functionalization through molecule structure tailoring $[14,15]$ and physical blending [16], which opens a model of boosting the product performance or creating product differentiation by changing the active materials instead of the manufacturing facilities. Last but not the least, with OSCs of different functionalities and also versatile device 
structures available for making OTFTs, different functions can be easily integrated with very similar processes on the same plastic substrate to create shatterproof flexible integrated systems in compact form factors with nearly no increase in thickness and weight.

In summary, the OTFT technology is able to bring electrical, mechanical and industrial benefits to many applications by activating non-flat surfaces with flexible displays, sensors and other electronic functions. The promising applications of OTFTs thus cross many markets including consumer electronics (e.g. wearables and smart home), automotive, security (e.g. flexible fingerprint sensors for mobile, smart cards, and beyond), and medical industry (e.g. low cost unbreakable X-ray imagers). As predicted by IDTechEx, the overall market for flexible electronics is forecast to reach nearly $\$ 70$ Bn by 2026 Due to their inherent amplification functions, good biocompatibility, high mechanical flexibility, and ease of miniaturization/integration, OTFTs have also emerged as a versatile platform for physical, chemical and biological sensing applications [17, 18, 19]. Motivated by these, in last decades, significant efforts have been devoted to research and development of OTFT technologies, including materials, processes, devices, circuit integration and applications, resulting in massive progresses [20]. The reported mobility values of OTFTs are much higher than a-Si TFTs, and even comparable with AOS and LTPS TFTs [21, 22]. Several material companies, including Merck, BASF, Polyera, NeuDrive and Smartkem, can supply a commercial grade stack of OSC, dielectric and interfacial materials for OTFTs. Lots of interesting advances in device engineering, circuit integration and applications of OTFTs have also been reported by different research groups and companies [23-28].

However, despite both strong application demand and these technological advances, there is still a gap to be filled for the OTFT technology to be commercially adopted. This paper will provide a comprehensive review of the current status of OTFT technologies ranging from material, device, process, integration to design and system applications, and clarify the real challenges behind to be addressed to enable the whole community to work together to find the solutions.

\section{REVIEW OF CURRENT STATUS}

There have been several good review articles on OTFTs published before, which are mainly from perspectives of fundamental material research and understanding related carrier transport and modulation physics [20, 29-38]. This part provides a comprehensive review of the current status of OTFT technologies ranging from material, device, process, integration to design and system applications.

\section{A. Materials}

\section{1) Organic semiconductors}

OSC is vital to OTFT, since it accommodates charge carrier to transport inside and thus mainly determines device performance. They can be synthesized with various functional groups to attain specific electrical and/or chemical properties. Consequently, a large number of OSCs have been developed $[35,36]$, as summarized in Fig. 1 . OSCs can be processed by either physical vapor deposition or solution-based processes. Whilst lots of academic research work used thermal evaporation to understand the fundamentals of charge transport and device physics, test the materials, or demonstrate the application ideas, industrial focus was on solution processing to provide low cost and scalable manufacturing options [9]. In general, the majority of OSCs are p-type semiconductors for both small molecules and polymers as seen in Fig. 1. One reason is that the electron-injection barrier is much higher than that of hole injection because the work function of accessible materials for electrode use is high and the OSC band gap is usually large [37, 38]. Another reason is that electron transport is prone to charge trapping.

In the 1990s, the highest performing soluble OSC materials were conjugated polymers, based on regio-regular poly (3-hexylthiophene-2,5-diyl) (P3HT) $\quad(\mu=0.01$ to 0.1 $\left.\mathrm{cm}^{2} \mathrm{~V}^{-1} \mathrm{~s}^{-1}\right)$ [39], whereas lower mobility but more environmentally stable polytriarylamines (PTAA) $(\mu=0.001$ $\mathrm{cm}^{2} \mathrm{~V}^{-1} \mathrm{~s}^{-1}$ were developed from chemistries originating in the organic photoconductor industry [40]. Other polymer based OSCs, such as region-regular polyquaterthiophenes (PQTs) [41], poly(2,5-bis(3-alkylthiophen-2-yl)thieno(3,2-b)thiophene) (PBTTT) [42], poly(9,9-din-octylfluorene- alt -benzothiadiazole) (F8BT) [43], have extensively been studied for OTFTs. However, the general issue with polymer OSC was the low mobility, which was difficult to exceed $1 \mathrm{~cm}^{2} \mathrm{~V}^{-1} \mathrm{~s}^{-1}$. Lately, donor-acceptor (D-A) copolymers, such as cyclopentadithiophene-benzothiadiazole (CDT-BTZ) [44], indacenodithiophene-co-benzothiadiazole (IDT-BT) [45], broke such a convention and exhibited remarkably high mobility more than $1 \mathrm{~cm}^{2} \mathrm{~V}^{-1} \mathrm{~s}^{-1}$. It was found that the charge transport in this family of polymeric OSCs (e.g., in IDT-BT) is no longer limited by $\pi-\pi$ stacking among molecular chains that often necessitates tight molecular packing motif and thus sacrifices the benefits of quasi-amorphous polymer. Rather, the aggregates formed by disordered sub-domains interconnecting to enable macroscopic charge transport in electronic devices once the molecular weight is high enough, and the charge transport observed is approaching disorder-free levels [46].

The other type of soluble OSCs is based on small molecules, enabling the benefit of higher mobilities than polymeric OSCs. Typical materials include triisopropyl-silylethynyl pentacene (TIPS-pentacene)

[47], 2,8-difluoro-5,11-bis(triethylsilylethynyl) anthradithiophene (diF-TESADT) [48], 2,7-dioctyl[1]benzothieno[3,2-b][1] benzothiophene (C8-BTBT) and the relevant derivatives (Cn-BTBT) [21, 49, 50]. Though high mobility and band-like charge transport can be obtained [51], the polycrystalline or single-crystal small-molecule OSCs are sensitive to microstructural defects created by impurities and mechanical strain, and hence they are not well suited for manufacturing large-area flexible electronics with ease of processing, and good uniformity. To solve this issue, a blend of small molecule acene semiconductor such as TIPS pentacene and diF-TES-ADT with low permittivity (low- $k$ ) polymeric binders was developed to improve crystallization control for higher carrier mobility and better uniformity $[16,52]$. Interestingly, a blend of small molecule OSCs with high-k binder formulation has also demonstrated excellent uniformity of high mobility $(\mu>4$ 
$\mathrm{cm}^{2} \mathrm{~V}^{-1} \mathrm{~s}^{-1}, \pm 5 \%$ ), and very importantly at a relatively short channel length of $10 \mu \mathrm{m}$ [53]. Recently, extraordinarily high mobility values of several tens of $\mathrm{cm}^{2} \mathrm{~V}^{-1} \mathrm{~s}^{-1}$ have been reported for p-type OSCS, for both polymer and small molecules, as seen in Fig. 1, indicating the great potential for performance improvement with OSCs through chemical structure design and process optimization [21, 22]. However, these high mobility values are shown to be significantly overestimated because the mobility extraction is based on the ideal FET model while neglecting the deviation of the electrical behavior of the fabricated OTFTs from that of an ideal FET [54].

To achieve complementary type circuits with large noise immunity and low static leakage current, the development of n-type OSC with performance comparable to p-type materials has been the main research focus $[55,56,57]$. For $n$-type OSCs, typical examples are fullerene $\left(\mathrm{C}_{60}\right)$ [58] and its sister $\mathrm{C}_{70}$ [59], as well as the newly developed poly \{[N,N0-bis(2-octyldodecyl)-naphthalene-1,4,5,8-bis(dicarboxi mide)-2,6-diyl]-alt- 5,50-(2,20-bithiophene) \}(P(NDI2OD-T2), also N2200) [60]. The highest electron mobility observed so far was about $1.2 \mathrm{~cm}^{2} \mathrm{~V}^{-1} \mathrm{~s}^{-1}$ in small-molecule NDI3HU-DTYM2 [61] and $6.4 \mathrm{~cm}^{2} \mathrm{~V}^{-1} \mathrm{~s}^{-1}$ in polymeric N2200 processed by bar coating [62].

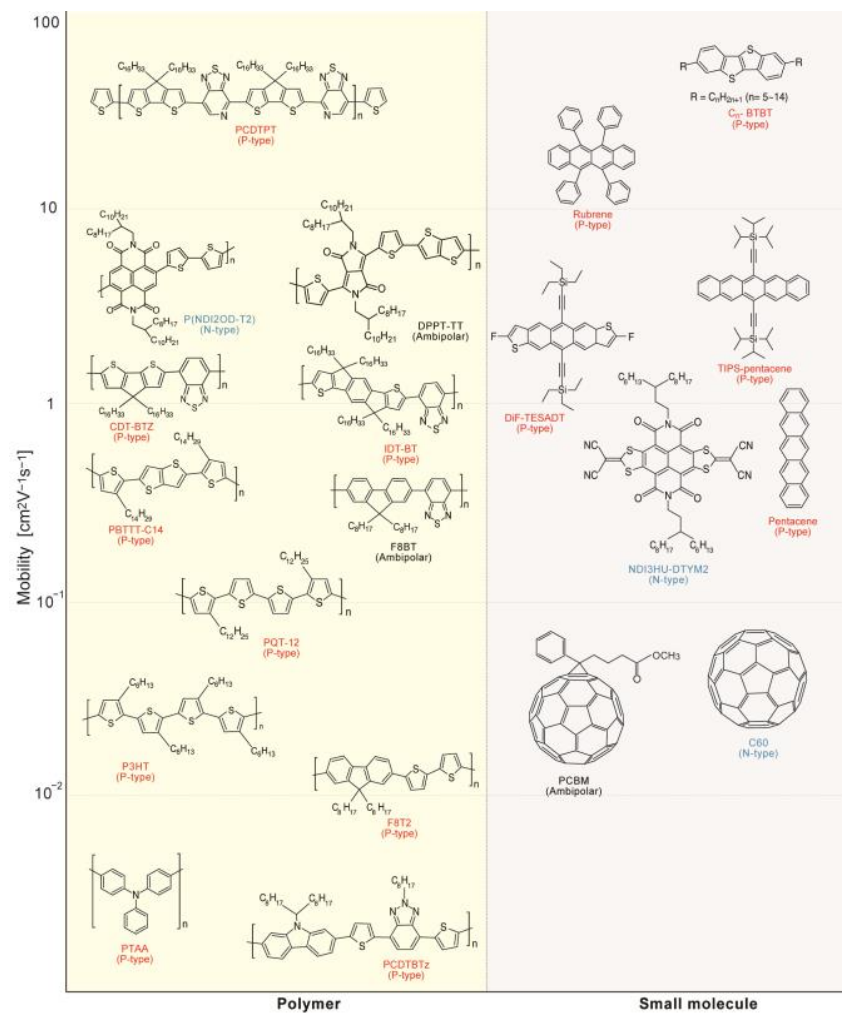

Fig. 1 Summary of typical organic semiconductors for OTFT applications arranged with respect to the carrier mobility, where the OSCs are classified as polymer and small molecule. Also, their typical transport type is indicated below the abbreviation. It must be noted that those reported very high mobility values are shown to be significantly overestimated because the mobility extraction is based on the ideal FET model while neglecting the deviation of the electrical behavior of the fabricated OTFTs from that of an ideal FET [54].

\section{2) Gate Dielectrics}

Gate dielectric is another vital component in OTFTs since it serves as a barrier between the charge carriers transporting at the semiconductor-dielectric interface and those in the gate, and hence it significantly affects OTFT performance. Gate dielectric determines the areal dielectric capacitance $\left(C_{i}=k \varepsilon_{0} / t_{i}\right)$ and accordingly the areal charge density in the channel $\left(Q_{i}\right)$, where $k$ is the dielectric constant relative to that of vacuum $\left(\varepsilon_{0}\right)$ and $t_{i}$ is the dielectric thickness. A large $C_{\mathrm{i}}$ is desired for reducing the operating voltage, enhancing the transconductance and minimizing the short-channel effects with scaled devices [63]. It can be obtained by either decreasing $t_{i}$ or is increasing $k$. For the former, however, the gate leakage should not be increased and for the later the device performance cannot be sacrificed.

Table II Summary of typical polymer gate dielectrics utilized for
\begin{tabular}{|c|c|c|}
\hline Dielectric & $\begin{array}{c}\text { Dielectric } \\
\text { constant }\end{array}$ & Reference \\
\hline CYTOP & 2.1 & {$[40]$} \\
\hline Polystyrene & 2.6 & {$[68]$} \\
\hline BCB & 2.65 & {$[69]$} \\
\hline Polyimide & 3.4 & {$[70]$} \\
\hline PVC & 3.4 & {$[71]$} \\
\hline PMMA & 3.5 & {$[40]$} \\
\hline SU8 & 3.9 & {$[72]$} \\
\hline PVP & 4.5 & {$[73]$} \\
\hline PVA & $7.8-10$ & {$[74]$} \\
\hline CYEL & 12 & {$[75]$} \\
\hline PVDF-TrFE & - & {$[76]$} \\
\hline PVDF-TrFE-CTFE & $>60$ & {$[78]$} \\
\hline
\end{tabular}

In the literature, many OTFT research activities used commercially available thermal oxidized $\mathrm{SiO}_{2}$ as the gate dielectric, with a self-assembled monolayer (SAM) treatment to suppress the influence of hydroxyl groups on OSC molecular packing and carrier transport $[31,64]$. Several high- $k$ inorganic dielectrics were also employed [64, 65]. However, from an industry perspective, to achieve a commercially competitive OTFT technology, solution processable polymer dielectrics are preferable, because of their potential for low cost manufacturing and excellent mechanical flexibility, though some vacuum deposited organic dielectrics have also been studied to provide high insulation properties [66, 67]. Various solution processable polymer dielectrics (e.g., CYTOP [40], polystyrene [68], BCB [69], polyimide (PI) [70], PVC [71], poly (methyl methacrylate) (PMMA) [40], SU8 [72], polyvinylphenol (PVP) [73], and poly(vinyl alcohol) (PVA) [74]) have thus been extensively studied as summarized in Table II. However, most polymeric dielectrics have relatively low $k$. As a result, with a thick dielectric layer required to eliminate gate leakage and be compatible for large area solution based processing, the reported OTFTs often require an operation voltage of a few tens of volts or even above $100 \mathrm{~V}$.

High-k dielectrics could help to achieve low operation voltage with a thicker layer. However, there are very few choices of suitable polymer dielectrics with high enough $\mathrm{k}$ values. Cyanoethylpullulan (CYEPL) [75], ferroelectric polymer poly (vinylidenefluoride-trifluoroethylene) (P(VDF-TrFE)) [76] and relaxor ferroelectric polymer poly(vinylidene fluoridetrifluoroethylene-chlorofloroethylene) (P(VDF-TrFE-CFE) $[77,78]$ have been studied for low voltage OTFTs. $\mathrm{P}(\mathrm{VDF}-\mathrm{TrFE}-\mathrm{CFE})$ was reported to have a $k$ value above 60 [78]. When the high-k gate dielectric layer is directly interfacing the OSC channel, the energetic disorder caused by the dipoles in high- $k$ dielectric would tend to trap carriers from gate bias induced conduction channel. The resulted localization 
of charge carriers could cause not only mobility degradation [40], but also increased hysteresis and device instabilities [79]. It was shown that, by inserting a thin, low- $k$ dielectric layer between the high- $k$ one and the OSC channel, the dipole field can be effectively screened for improved device performance [79]. In [80], it was found that, during spin-coating of a blend of high molecule weight PMMA with P(VDF-TrFE), the high molecule-weight PMMA tended to aggregate at the bottom of the deposited film, thus forming the low- $k$ /high- $k$ bi-layer gate dielectric structure for OTFTs in a simpler way.

A type of solid polymer electrolytes, so-called ion gels, has also been studied as a gate dielectric for low voltage OTFTs [81, 82]. The ion gel was obtained by blending ionic liquids with a gelating triblock copolymer to form a physically cross-linked network and features very large specific capacitance exceeding $1 \mu \mathrm{F} / \mathrm{cm}^{2}$ with thickness of about $1 \mu \mathrm{m}$, so that it can help to substantially reduce the operation voltage and simultaneously achieve very high driving currents. Despite that, the long polarization time of the ion gel dielectric may slow down the device operating speed, and the presence of ions in the solid electrolyte can cause electrochemical doping of the channel, thus inducing reliability issues.

Furthermore, in the fabrication of OTFTs devices, the deposition of two or more layers sequentially by solution processes could cause dissolution or swelling of the underlayer. Cross-linking of the polymer dielectrics by photo- or thermal reactions have been studied to enhance solvent resistance, and also improve the electrical robustness [83-87].

\section{3) Other Materials}

With significant advances in fundamental material development, the industry can now supply volumes of OSC and gate dielectric materials for substantial pilot scale manufacturing processes of low temperature flexible display backplanes with the device performance greater than a-Si TFTs. These materials have either been commercially available materials, which have been evaluated for compatibility or, in some cases, new materials have been synthesized with tailored properties. In addition to OSC and gate dielectric materials, other materials for substrate buffer layers, electrode treatment and passivation layers are also of importance. In the multi-layer integration structure, materials at different layers have to be well matched with solubility difference or be cross-linkable. The OSC/dielectric interface is a critical one for efficient charge transport, as is the back-channel of the OSC layer. Therefore, in a top gate device, it is necessary to control the buffer layer [88], while in a bottom gate device, selecting a suitable passivation layer is critical [89]. Additionally, metallic source/drain (S/D) electrodes often require SAM treatment to improve charge injection into the OSC layer [31]. The S/D electrodes used in manufacturing as opposed to research have to be compatible with the industry available processes and at an acceptable cost. Alternatives to gold $\mathrm{(Au})$ are vital to the success of the industry, with silver (Ag) [74], indium tin oxide (ITO) [90], molybdenum (Mo) [91] and copper ( $\mathrm{Cu}$ ) [92] being amongst the list of some that have been investigated for this purpose. Therefore, the industry needs to work on developing a full package of materials alongside the OSCs to provide a total material solution for OTFT manufacturing.

\section{Device Structure}

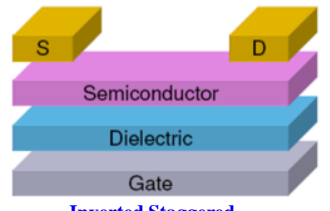

Inverted Staggered
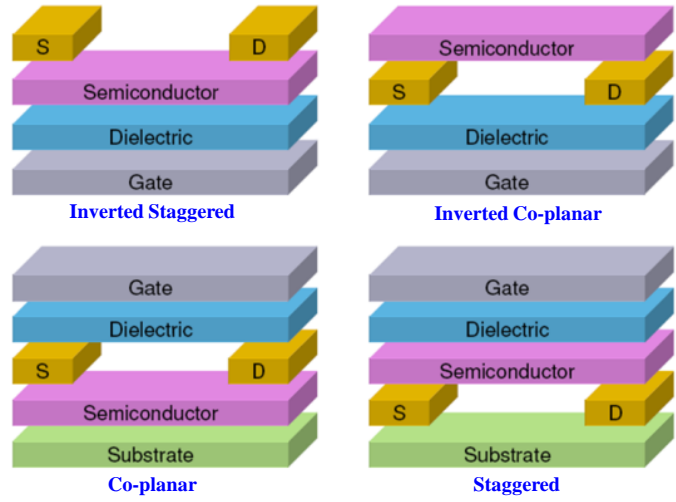

Inverted Co-planar

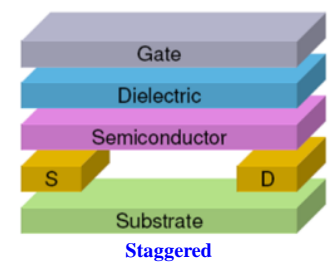

Fig. 2 Illustration of the four device structures used for OTFTs.

As shown in Fig.2, OTFTs can be implemented in four different structures depending on the relative locations of the electrodes. For most of basic research, the inverted staggered structure is used with highly doped silicon as the gate and thermally oxidized silicon oxide as the gate dielectric based on commercial silicon wafers for process simplicity [31]. Many reported high mobility OTFTs were also fabricated in this way $[15,21,22]$. The co-planar structure was also recently realized to obtain excellent field effect transistor characteristics for ambipolar polymer OSCs by selective contact doping using p-type dopants [93]. However, these top-contact structures are difficult for OTFT circuit integration over large area because of the process difficulty of making S/D metal electrodes on top of the OSC layer with precise patterning. For bottom contact structures (inverted coplanar and staggered), the metal contacts are formed before deposition of the OSC layer, and can thus be finely patterned by industry compatible photolithography processes. Surface modifications of metal contacts with thiol SAMs can be used for obtaining low resistive and preferably ohmic metal/semiconductor contacts as well as good-quality semiconductor films on top of the S/D electrodes for higher performance and better uniformity of electrical characteristics [94]. With these considerations, they are also the choices in OTFT manufacturing trials in industry $[95,96]$. Compared to the inverted coplanar structure, the staggered structure has a lower contact resistance for a larger effective area for carriers' injection at the source electrode. The contact resistance in the staggered structure OTFT was also found to be less dependent on the contact barrier than that in the inverted coplanar one, attributed to gate field enhanced charge injection [97].

Contact effects are usually present in OTFTs and they manifest by a reduction of effective mobility, and transconductance. For this reason, scaling of channel lengths may be problematic, as the limiting effect of the contact becomes more pronounced. However, deliberately introducing a contact energy barrier may have beneficial effects despite the decrease in current and speed. A new structure named source-gated transistor (SGT) [98] is formed when a barrier (e.g. Schottky) at the source is used to restrict the current. Under normal operation, at low drain bias, the source barrier is reverse biased and the semiconductor is depleted of carriers across its whole thickness, leading to saturation of output current at comparatively low drain voltage. This allows energy-efficient 
operation while maintaining high intrinsic gain, from lower drain voltages than conventional FETs, and current is modulated in the first order by the gate electric field's effect on the effective source barrier height [99]. When this structure is applied for OSCs, the induced high drain electrical field in the device may result in enhanced carrier mobility and operating speed [100]. As a result of the current control method, drain current is virtually independent of source-drain separation, which increases performance uniformity in high throughput technologies with low resolution patterning. Importantly, the SGT structure was normally realized in inverted staggered structure, and would also possible to be made in staggered structure. Therefore, SGT and the conventional FET can be made on the same substrate, by varying the contact properties through choice of a different electrode metal or contact treatment, enabling the exploitation of both types of devices in the same circuits.

\section{Process and Integration}

The most ideal approach for OTFT integration is full additive printing for "manufacturing-on-demand" at a fraction of the cost and footprint. However, due to difficulties in technology scaling for fine structures and complicated multi-layer integration, it is unrealistic today to use all additive printing processes for high end applications, such as displays, which have strict requirements on performance, integration density and reliability. Thus, it's clear in the short term, OTFT based flexible electronics must be manufactured using more conventional processes - by repurposing existing manufacturing equipment to minimize barriers to entry and get products to market that validate the 'product performance' and applications and prove business cases for the value that OTFT can bring to applications. In the meantime, lots of research is undergoing on addressing issues on all printing processes, for many very cost sensitive applications with less requirements on the integration density and reliability.

\section{1) Processes compatible with established industry facilities}

The display industry currently manufactures over 150 million square meter of display backplanes per year on glass, which is supported by mature industry players ranging from materials suppliers to equipment vendors. In the near future OTFT can access some of this capacity for production of plastic based displays and other applications provided that the materials are compatible with established toolsets. A non-exhaustive list of reported examples of OTFT processes demonstrated at pilot manufacturing scale include (substrate size in brackets): FlexEnable in the UK (14 inch square), Sony in Japan (6 inch round), LG Display in South Korea (370 $\mathrm{mm} \times 470 \mathrm{~mm})$, Samsung $(300 \mathrm{~mm} \times 400 \mathrm{~mm})$, Polyera in Taiwan (200 $\mathrm{mm}$ square) and CPI in the UK $(200 \mathrm{~mm})$. The Plastic Logic manufacturing facility in Germany (Gen 3.5, $650 \mathrm{~mm} \times 780 \mathrm{~mm}$ ) based on the FlexEnable process is currently the only example of a full manufacturing line for OTFTs. The processes currently employed in the display industry and also used in OTFT manufacture include sputtering, photolithography, wet etching, dry etching, spin coating and slot die coating. Materials used for the OSC layer are perhaps the most sensitive and exposure to oxidizing chemicals such as nitric acid and aggressive organic solvents such as photoresist stripper should be avoided throughout the process. Sputtering and dry etching have also been reported to have impacts on the OTFT characteristics of certain materials [101-103]. Some degree of protection is thus often required by dielectric, metal or other photoresist layers throughout the processes so that aggressive chemicals and processes can be used. With the high resolution patterning, good overlay accuracy and low defect density common to display manufacturing, OTFT can achieve high performance with good uniformity in prototype devices, examples being rollable OLED displays [104], production quality electrophoretic products [105] and high frequency (1 $\mathrm{MHz}$ ) logic, as shown in Fig. 3.

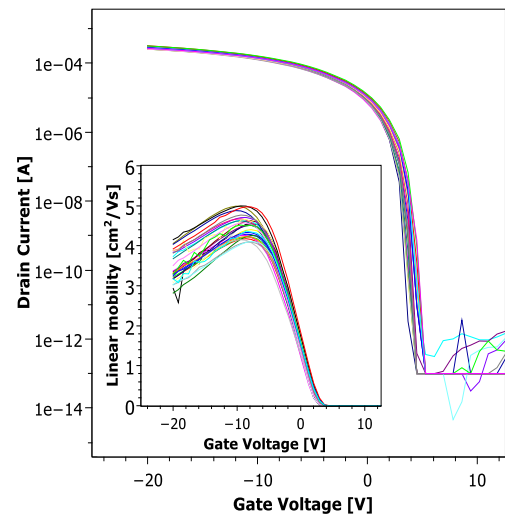

(a)

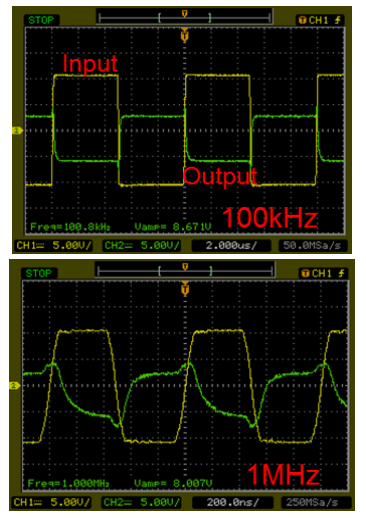

(b)
Fig. 3 (a) Transfer curves for OTFTs made using FlexOS ${ }^{\mathrm{TM}}$ OSC in staggered Corbino structure with a channel length of $7 \mu \mathrm{m}\left(V_{\mathrm{DS}}=-2 \mathrm{~V}\right)$, and the measured linear mobility average was $4.4 \mathrm{~cm}^{2} / \mathrm{Vs}$ for 30 devices with standard deviation of 7\% as shown in the inset; (b) Transient characterization of NAND3 logic gate made with the OTFT at $100 \mathrm{kHz}$ and $1 \mathrm{MHz}$ driven from a function generator and with power supply voltage of $-20 \mathrm{~V}$.

\section{2) Full printing processes}

Besides being developed for various flexible backplanes for e-paper, LCDs, OLED displays and imagers, OTFTs can find strong opportunities in the short/medium term for mass produced disposable electronic systems so called "smart labels" or "smart tags", which are indeed not economically viable to be realized by the established silicon technology.
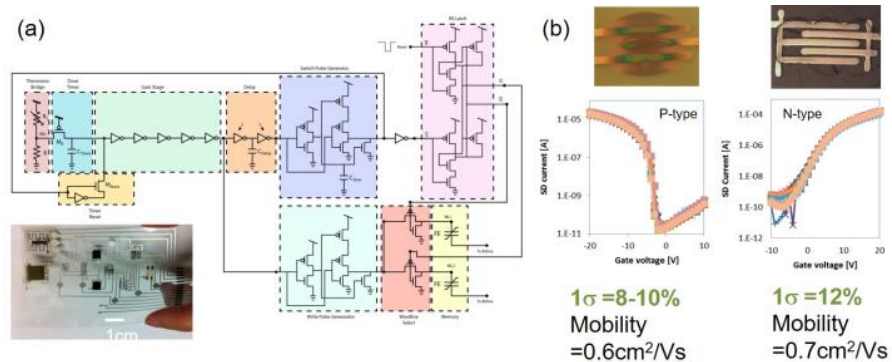

Fig. 4. (a) Printed time-temperature dose tag showing the different sub-circuits and a photograph of the tag. Reprint with permission from [107]. (b) For this tag, the transfer characteristics for 30 typical OTFTs using p-type semiconductor or n-type semiconductor. The photographs show the semiconductors printed over interdigitated electrodes.

The simplest applications are based on the all organic stand-alone system. For example, a low-scale of integration logic block was connected to a suitable sensor, i.e. a toggle 
button and/or a simple fuse, to detect the opening of a package, and can drive a simple display showing the status of such package [106]. Such simple, stand-alone, yet effective tag, could be attached to the package in the same way a normal graphical label is, sharing a very similar geometrical form factor and mechanical properties, provided that a suitable interconnection to the sensor is guaranteed. Such possibility must however be sustained by the development of high-throughput, yet reliable printing processes, capable of keeping the overall tag cost extremely low. As shown in Fig. 4 (a), a time-temperature dose tag was fabricated on flexible plastic foil and comprised a thermistor divider, complementary organic circuits, and two nonvolatile memory cells [107], after achieving having reasonable device uniformity of both p-type and n-type OTFTs with additive printing processes (Fig. 4(b)). If integrated with an appropriate power supply, the tag could operate as a stand-alone system, with nonvolatile memory for later readout for a wide range of potential applications in food and medicine spoilage. It is a promising step towards economical printed OTFT stand-alone sensor systems.

For the commercially available batteries used as the power supply for these stand-alone systems, the output voltage level of around $1.5 \mathrm{~V}-4 \mathrm{~V}$ is often insufficient to surpass the threshold voltage for many printed OTFTs that have been demonstrated so far. In order to operate the system, either the supply voltage must be increased or the operation voltage of OTFTs should be reduced. The supply voltage can be boosted by connecting batteries in series or by adding a voltage multiplier [108] or charge pump circuit [109], which, however, increases the system complexity and cost. Low voltage operable and stable OTFT is thus very important for this system, but it is challenging to be achieved by only enlarging the gate dielectric capacitance with ultra-thin or high- $k$ gate dielectric layer, especially when the devices need to be manufactured by all additive printing processes to make a commercially viable technology. Recently, several studies have shown that it is feasible to reduce the sub-gap density of states (DOS) at the channel through solution processed organic semiconductors for realizing low voltage OTFTs with small gate dielectric capacitance $[74,110]$. Therefore, a relatively thick low- $k$ polymer dielectric can be applied, which can help to achieve excellent stability and also has wide material choices $[71,72,83$, $86,87]$. Such a low voltage OTFT can be fabricated by inkjet printing all layers including electrodes, gate dielectric, OSC and encapsulation layers, proving the most economic manufacturing approach [111].

For more envisioned applications, which need to have wireless connections with users, the stand-alone system is not applicable. In such systems, a series of functions, including accurate and complex signal processing, power management and communication are required to be performed in a severely power constrained system. These functions are very challenging to be realized with printed OTFTs today. A hybrid technology is thus necessary to combine the OTFT with low voltage silicon chips [112]. In such a system as illustrated in Fig. 5, a transducer circuit is implemented using printed OTFTs to convert the sensed signal to a standard voltage output. Various functional sensing materials can also be integrated through the printed OTFT technology. A common read-out and signal processing hardware or specific silicon chip consisting of analog-to-digital converter, processor, wireless interface for data output (e.g. near field communication, Bluetooth, ...) can thus be implemented for these different sensors. With a fixed silicon chip design and additive printing of the OTFT based front end for the custom design, this hybrid integration would provide a low cost and versatile solution to "smart tag" systems. For this integration, With the fully-printable low voltage and stable OTFT, sensor tags were made to be operated in a low voltage $(3.3 \mathrm{~V})$ battery powered electronic system for long-term and repeatable ammonia and $\mathrm{pH}$ sensing, respectively [24, 71].

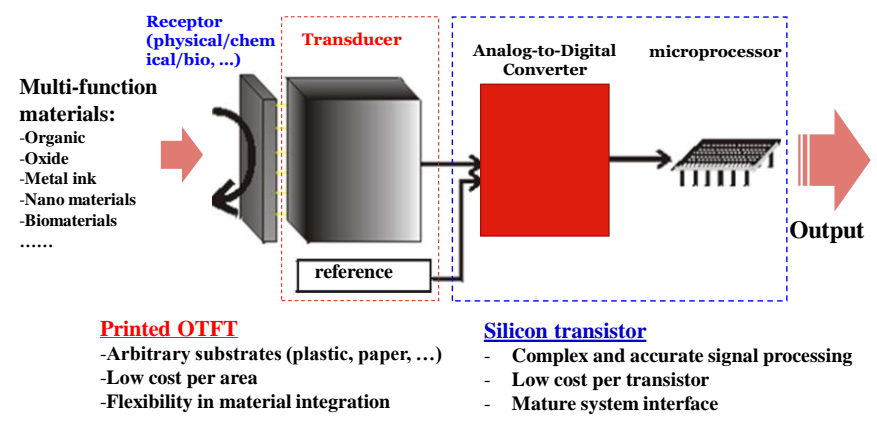

Fig.5 Illustration of a hybrid integration approach combining the advantages of both the printed OTFT and the silicon technology.

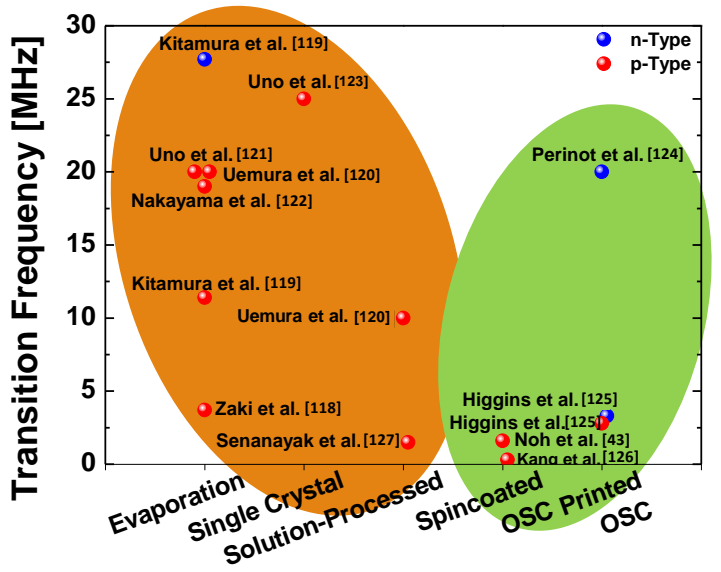

Fig. 6 Overall comparative plot of the best $f_{\mathrm{T}}$ results for OTFTs, which $f_{\mathrm{T}}$ were explicitly measured and reported in the literature. The works are classified with respect to the fabrication methods adopted for the patterning of the electrodes and for the deposition of the semiconductor.

Compared to the hybrid solution with a silicon chip, a "chipless" design would be desired to provide a lower cost solution for wireless sensor tags by seamlessly integrating all the required electronics, sensing and antenna driving functionalities [113, 114]. For that, high speed printed OTFTs to afford signal modulation frequency of at least tens of $\mathrm{MHz}$ are needed to construct the wireless communication interfaces. The transition frequency or cut-off frequency $f_{\mathrm{T}}$ is widely adopted for the evaluation of the operation speed of a single transistor [115-118]. Two methods were generally adopted for 
measuring $f_{\mathrm{T}}$ : a direct evaluation of the transconductance and gate capacitance via the measurement of small current signals flowing into the device electrodes $[116,117]$ or the evaluation of the two-port-network $h_{21}$ hybrid parameter [118]. Enhancing $f_{\mathrm{T}}$ of OTFTs requires the adoption of OSC materials guaranteeing high effective mobility and architectures with scaled channel lengths and reduction of parasitic capacitances posing constraints on the patterning technology that has to be maintained compatible with cost-effective large-area processes. Fig. 6 summarizes the measured $f_{\mathrm{T}}$ of OTFTs from selected works achieving the best results, which are classified with respect to the fabrication methods adopted for the patterning of the electrodes and for the deposition of the OSC layer [43, 118 -127]. The record $f_{\mathrm{T}}$ reported to date reaches the value of 27.7 $\mathrm{MHz}$ for a device based on $\mathrm{C}_{60}$ with a channel length of $2 \mu \mathrm{m}$ defined by photolithography [119]. Recently, OTFTs with $f_{\mathrm{T}}$ of $20 \mathrm{MHz}$ were fabricated by means only of scalable coating techniques and laser-based direct-writing methods with a completely mask-less procedure [124]. A much higher throughput approach was demonstrated by Kang et al. [126], who used highly-scaled gravure printing to fabricate OTFTs with a fast printing speed of $1 \mathrm{~m} / \mathrm{s}$, despite a lower $f_{\mathrm{T}}=0.3 \mathrm{MHz}$. It is important to highlight that those high $f_{\mathrm{T}}$ values were generally achieved with operation voltages of several tens of volts. Indeed, to comply with the requirement of low-voltage operation which is necessary for applications in the fields of wearable and portable devices, it is desirable to achieve high-frequency operation for a supply voltage lower than such a value. The works where high-frequency operation is combined with low-voltage operation are a minority. The representative work by Zaki et al. obtained $f_{t}=3.7 \mathrm{MHz}$ for a bias voltage as low as $3 \mathrm{~V}$ [118].

\section{E. Device Modeling and Circuit Design}

Accurate compact models are basis for efficient circuit design to move the OTFT technology from devices to circuits and systems. In the past, corresponding development of physics-based compact models has been under continuous progress, after many fundamental aspects of OTFTs were revealed [128, 129]. Models to be able to capture non-ideal realistic device behaviors, including gate bias $\left(V_{\mathrm{GS}}\right)$ dependent mobility [130, 131], non-exponential dependence of subthreshold current on $V_{\mathrm{GS}}[132,133]$, and contact resistance effects [134], have been developed. Smooth linear-to-saturation transition and subthreshold to above-threshold transition was further worked out to connect these discrete models for unified compact models, which covers all regimes of OTFT operations and are able to be implemented into circuit simulators to reach an effective convergence $[130,135]$. For the large variations in OTFT materials and structures, further modeling efforts have been devoted to the capability of rearrangement and flexibility for capturing the essence in the behaviors of various OTFTs in a consistent and relatively simple way, and allowing for easier implementation, modifications and parameter extraction [128, 136]. These developed models can be conveniently implemented into commercial circuit simulator for circuit simulation using Verilog-A, which, however, is slower and less efficient than a well-developed SPICE model [137]. Currently, the only OTFT SPICE model available in commercial circuit simulator is the universal OTFT (UOTFT) model in SmartSPICE, which combines the robust concepts of universal charge-based FET modeling with OTFT specific charge, mobility and contact resistance bias and temperature dependence [138]. The parameters in the UOTFT is significantly less than that in those advanced models for modern silicon FETs, as shown in Fig.7.

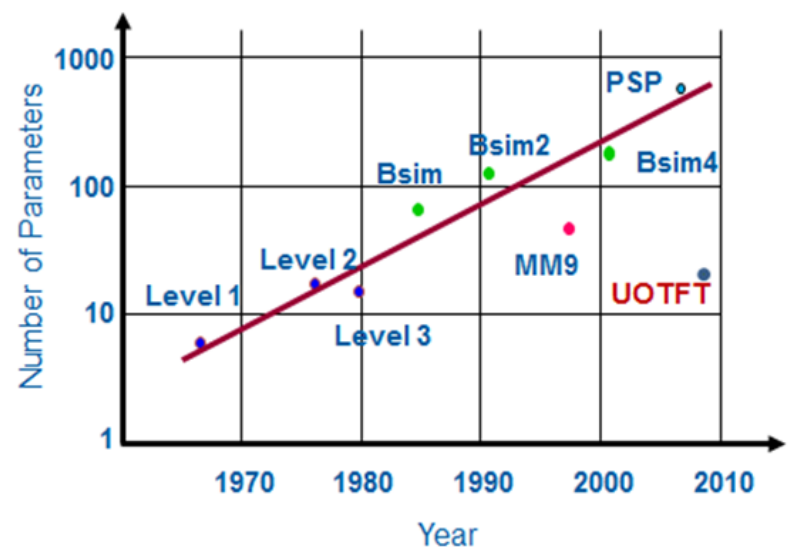

Fig.7 Comparison of the number of parameters in the developed universal OTFT model in commercial circuit simulator SmartSPICE with the models for silicon FETs.

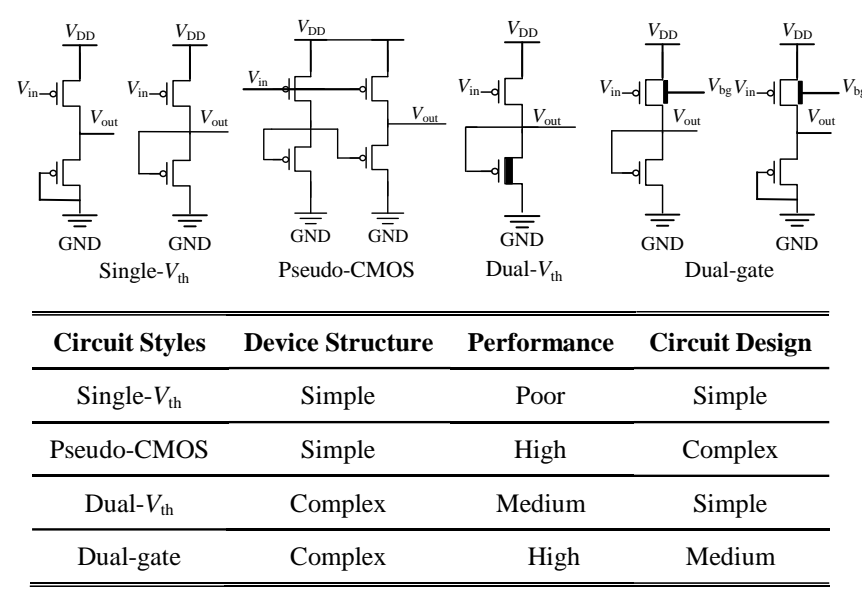

Fig. 8 Comparisons of different unipolar circuit styles for OTFTs.

To design and construct complex circuits and systems, the complementary type is a preferred choice for its low static power, rail-to-rail operation and large noise margin [139]. However, it is difficult to find $\mathrm{n}$ - and $\mathrm{p}$-type semiconductor materials with equivalent performance and compatible processes that would allow easy fabrication of high performance complementary circuits. For print processing, complementary design is easy to realize since the two semiconductors are additively patterned in just two steps. For photolithographic processing, at least three more masking steps are required to pattern two semiconductors due to the subtractive process. Therefore, the unipolar designs have been widely adopted for OTFT logic circuits. Although having the advantage of simplicity, the common unipolar logic circuit design could hardly provide sufficient noise margins to accommodate normal 
OTFT parameter variations for building complex circuits. To improve the robustness to parametric variability, advanced device or circuit architectures such as pseudo CMOS [140], dual- $V_{\text {th }}$ TFT logic [141], dual-gate structure [142] were proposed. Therefore, considering the tradeoff among performance, process simplicity and design complexity as shown in Fig. 8, it would be helpful to predict the yield and the performance in the early device development or circuit design stage to select the most cost-effective solution for realizing the required functional circuit of good manufacturability [143, 144].

\section{F. EDA tools and Design Kits}

Electronic Design Automation (EDA) tools have been a key factor for the success of the microelectronic industry since they allowed building complex (non-regular) integrated circuits with an increasing number of transistors (according the Moore's Law) in a reduce amount of time. EDA tools strongly developed the concept of technology-independent circuit design that is later mapped into technology dependent cells and structures (based on transistor models and design rules), that were described in the Technology or Process Design Kit (TDK or PDK) available for every stable technological process. This leads to the concept of fab-only and fabless semiconductor industry. Fab-only consists of companies that does not produce semiconductor products or chips being commercialized (i.e. TSMC or UMC) while fab-less companies are concentrated in application-oriented technology-independent that do not own fabrication processes (i.e. Qualcomm or ARM both around the mobile industry).

As shown in previous sections, design of future flexible systems based on OTFTs will include heterogeneous integration of different technologies (flexible + silicon, flexible + flexible, flexible + functionalization). Moreover, there is also an increase of homogeneous integration systems (displays + logic + actuators, sensors + processing + antenna) in a given technology and substrate. Both approaches require the implementation of complex integrated functionalities that required mixing different design disciplines and methods. Currently, there isn't any single EDA tool capable of coping all those aspects that require not only the corresponding device modelling but also mixed-mode simulators, variability analysis and detailed layout extraction tools.

Big players in the EDA industry such as Synopsys, Cadence or Mentor Graphics can cover almost all design aspects. Their tools allow very high design productivity at the cost of expensive licenses. Part of this design productivity comes from the fact that any given circuit or structure can be easily and quickly redesigned for a different technology or process variation thus strongly reducing circuit any redesign effort. Technology dependent tools are ready to use and the methods to add the information related to any OTFT or general TFT technologies are specified around the PDK concept [145]. However, those big players are not yet paying attention to specific tools for OTFT or TFT based systems. The main reason is that the process technology has a very wide range of choices, while existing valued market applications is much less compared to that of the silicon microelectronics industry as illustrated in Fig. 9.

Other EDA tools used for OTFT circuit design are: (1) other low cost PC-based EDA tools (i.e. Tanner or PhoenixBV), (2) tools coming from the display industry (i.e. Silvaco) that include a strong link with device level simulation, (3) the large set of tools coming from the PCB industry that are weekly connected with simulation but allow checking geometrical and electrical design rules before sending the circuit for fabrication, and are much cheaper; (5) open-source or free tools (i.e. Glade, Klayout, Alliance) and (6) there are still a high number of designs that are just being designed with graphic editors.

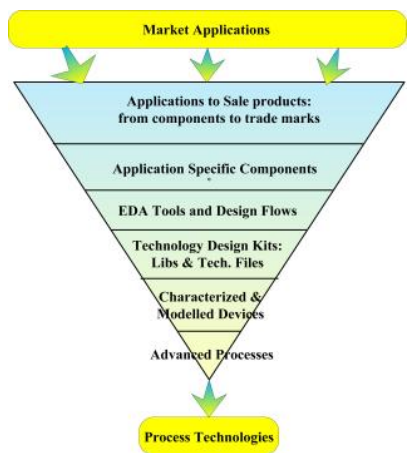

(a)

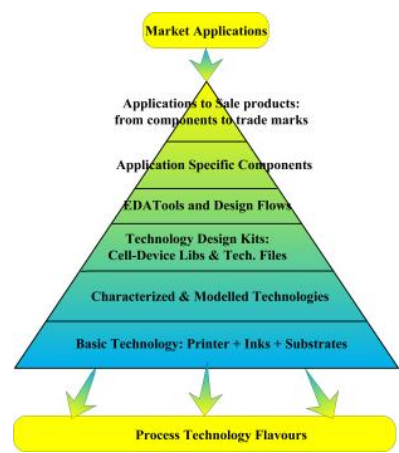

(b)
Fig. 9 (a) View of the current silicon microelectronics industry where many fabless industries develop a wide range of products implemented in a reduced set of fab facilities thanks to its highly segmented horizontal business model; (b) View of the current flexible electronics industry with a reduced set of products that have a wide range of technologies available for its implementation in a closed vertical business model.

EDA tools are usually related with design methodologies that have been traditionally classified as device-based (or full-custom) and cell-based (or semi-custom). Current OTFT circuits require low design effort compared with silicon ones [146]. Most of the implemented designs are still device-based $[23,147,148]$ so that designers and up by drawing the final circuit layouts. Nonetheless, there are several circuits that are already been built using cell-based design techniques that include some degree of automation in the placement and routing, such as standard-cells or gate-arrays [149-151].

A novel circuit design initiative is devoted to let designers use the capabilities of digital printing on top of TFTs built with other technologies for building circuits to improve their functional or performance characteristics by adding identification codes, calibration circuits, programmable memory or ad-hoc arithmetic circuits. Examples are: (1) user-customizable logic paper (UCLP) with sea-of-transmission-gates (SOTG) of organic CMOS and inkjet printed interconnects [151]; (2) Programmable Logic Circuits (PAL) for functional integrated smart plastic systems [152], (3) Print-Programmable Read-Only Memory (P2ROM) [153] and (4) Inkjet-configurable Gate Arrays (IGA) with high resolution OTFTs and inkjet printed interconnecting dots [154].

\section{G. Applications and Status of Industrialization}




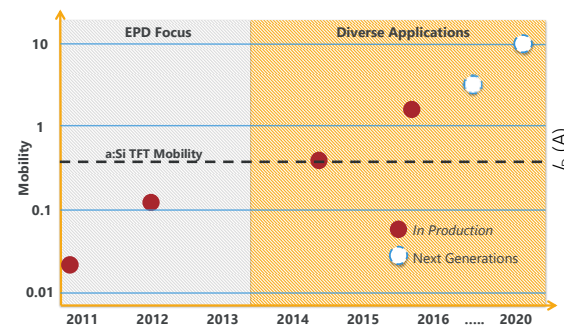

(a)

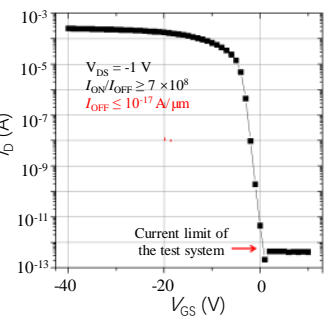

(b)
Fig. 10 (a) The performance roadmap in terms of mobility for the OTFT technology in FlexEnable; (b) The measured transfer performance of the current OTFT fabricated in FlexEnable with a channel length of $5 \mu \mathrm{m}$ and a channel width of $50,000 \mu \mathrm{m}$.

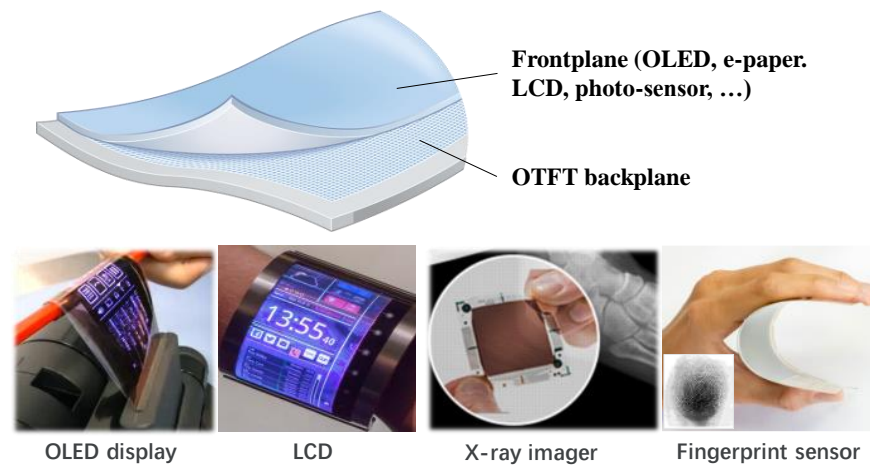

Fig. 11 Illustration of the integration structure and the photos of the developed flexible system applications based on the OTFT backplane technology in FlexEnable.

The OTFT backplane technology developed by FlexEnable (previously Plastic Logic) is the first and possibly only OTFT technology used in products today. The products are e-paper displays manufactured by Plastic Logic Germany (e.g. popSLATE, an iPhone cover containing a OTFT-driven flexible e-paper display). Within the past two years, the OTFT performance has risen to be above that of a-Si TFT for all key electrical parameters including mobility, stability and uniformity, opening up possibilities for a wide range of new applications. As shown in Fig.10 (a), the mobility of the OTFT is now about $1.5 \mathrm{~cm}^{2} \mathrm{~V}^{-1} \mathrm{~s}^{-1}$ at a channel length less than $5 \mu \mathrm{m}$. A separate but often less-discussed electrical benefit of OTFT is leakage current. Compared with a-Si TFTs, the OTFT can easily have leakage currents two or even three orders lower. Fig. 10(b) is the measured transfer curve of an OTFT with a channel width of $50,000 \mu \mathrm{m}$ in order to measure the leakage. It can be seen that even with such a large channel width, leakage is still lower than the noise in the test system - indicating very low true leakage current less than $10^{-17} \mathrm{~A} / \mu \mathrm{m}$ for the device. This very low leakage brings benefits to displays in allowing low-frame rate driving (power saving), and also to sensors, enabling greater sensitivity, which for applications like x-ray sensors means the use of lower doses. Besides the e-paper displays, a series of more advanced display and sensor applications have been demonstrated with this OTFT technology, as shown in Fig.11.

In addition to array backplanes, simple OTFT circuitry is promising for bio-sensor applications. Most of the OTFT-based biosensors, including organic field effect transistors (OFETs) and organic electrochemical transistors (OECTs), are potentiometric transducers [19]. A little change in the effective gate voltage will lead to a pronounced channel current response. Therefore, due to their inherent amplification functions, good biocompatibility, high mechanical flexibility, and ease of miniaturization/integration, OTFTs have emerged as a versatile platform for biological applications. Recently, different types of biosensors, including enzyme [155], DNA [156], cells [157], neuron [158], bacteria and protein sensors [159, 160], have been successfully developed based on the interactions between biological analytes and organic channel or gate electrode of an OTFT modified with specific biomolecules and functional materials. Although OTFT based biosensors have not yet been commercialized, some devices including enzyme sensors may find big market in the future. For examples, glucose sensor is a very important healthcare product since the population of diabetes mellitus in the world increases every year. The prevalence of diabetes for all age-groups worldwide was estimated to be $2.8 \%$ in 2000 and $4.4 \%$ in 2030 [161]. Currently, the operation of all of the blood glucose meters in the market is based on finger stick assays, which is painful and can cause stress to the patient. Therefore, developing noninvasive glucose meter with high accuracy becomes the trend of blood glucose determination. OTFT-based enzyme biosensors can be used for noninvasive glucose detection by measuring the glucose level in body fluid, which may have a huge market in the future [162]. Other enzyme biosensors, like uric acid and cholesterol sensors, are also very useful in healthcare products. Nevertheless, the commercialization of OTFT-based biosensors relies on the development of fabrication techniques for mass production and the synthesis of biocompatible and stable organic semiconductors specifically for biological applications.

\section{Challenges AND OutlooK}

The above review shows massive progresses on all aspects of the OTFT technologies. However, to become a widely adopted technology for the envisioned applications, there are still lots of challenges ahead to be addressed. This part will try to clarify these key challenges.

Firstly, in terms of materials, there is still lack of material stacks to be used for producing high performance, stable and uniform OTFTs in large area compatible processes. The current OTFT community is perhaps too focused on charge mobility of materials as a figure of merit. The processes and device structures used for testing the material performance are not for scalable manufacturing. A greater emphasis should be placed on other attributes such as uniformity, stability, manufacturability and short channel device performance. In addition, as highlighted by a recent paper, mobility estimation from non-ideal FET current voltage characteristics typical for OTFTs, have been shown to produce values that are incorrect by an order of magnitude or more [54]. When the high performance does not translate into the expected performance in the types of devices used in manufacturing, then this reduces confidence of the technology as a whole. Possibly the figures of merit for materials and devices should be defined by the electronic design community since they are the ones who will produce designs in the technology that meets the specifications of end users. Moreover, many physical and chemical interactions between the OSC layer and other dielectric, metal and interfacial layer 
materials in the device stack are not only important for initial device performance but are critical for control of the device stability under electrical stress [163]. In addition to performance, material stacks also need to be developed for processing OTFTs to be able to meet the strict reliability test requirements, which are defined by applications. Deep understanding of the instability issues of OTFTs and finding the solutions to develop stable and reliable OTFTs is thus of great importance [164].

The challenge with device uniformity have been the other main hurdle to practical large-scale OTFT circuits, especially when fabricated by printing [28, 107, 165, 166, 167]. The characteristics of printed OTFTs shows significant spread as shown in Fig. 4(b). For devices with the same nominal dimensions, the standard deviations of the transconductance is typically $<12 \%$. The device variations lead to decreasing circuit noise margin. To improve circuit yield, the devices need to be more uniform.

Secondly, there is no standard material stacks, device /integration architectures, and manufacturing processes for the whole community to work with. Diversity of materials choices, device structures, and processes are advantages of OTFTs to provide a versatile technology platform. However, it also brings challenges to the whole community to work together to address the common technology issues and build an industry chain towards commercialization like that in silicon semiconductor industry. For different types of applications, the processing approaches would vary to have the optimal balance between the performance and the cost. For example, for high resolution flexible display or imagers application, standard manufacturing and device integration architecture can be constructed based on the well-established processes in the display industry, including sputtering, photolithography, wet/dry etching, spin coating and slot-die coating. The large material research community can thus have clear objectives of matching the device integration and manufacturing processes, while achieving required performance. While the ongoing material and process research is valuable in tackling current and future device performance obstacles, the development of robust design tools require stable materials and processes. The development of industry standard OTFT compact models, and associated process/technology design tools is essential to lower the entry barrier for product designers. The design community will continuously add functionality and IP value which in turn leads to greater technology dissemination and adoption. Owning a strong design community will be able to speed up product development cycles, maximize the performance capability of the technology, and exploit the new applications based on the technology. Prerequisite to this is the development of manufacturing design rule manuals and standardized process design kits. Inbuilt in this are many challenges related to compact model parameter extraction templates, meaningful layout, design rule check tools and a seamless design automation flow.

An important advantage of OTFTs is that they can be manufactured by printing, eventually allowing a highly customized fabrication of organic and hybrid integrated circuits, especially when digital printing techniques are adopted, ultimately handing an on-demand electronics. This concept which has been developed at research level in the last one or two decades needs to face a reality where such tools that would allow this shift of paradigm in electronics fabrication, are not yet developed outside the laboratories. Therefore, printing OTFTs allowing design and custom fabrication of circuits needs more work on standardization of materials, components and processes, design tools and accessible fabrication tools.

The separation between fabs and fabless industries is popular for other mass market domains apart from the semiconductor industry (i.e. the traditional printing industry) but it is not for niche oriented domains (i.e. displays or MEMS). The integration of circuit with OTFTs seems to show that there will be room for mass production for a wide range of applications, as illustrated in Fig. 12. If this happens, fabs will open their technologies to the application-oriented industries by providing PDKs (geometric and simulation information) and clear cost models. Opening access to technologies will for sure boost and enlarge the applications since it will lower the entry barriers for those industries. Different initiatives have already set the basis for building this path [168, 169] and have demonstrated the feasibility of using this model to build complex systems.

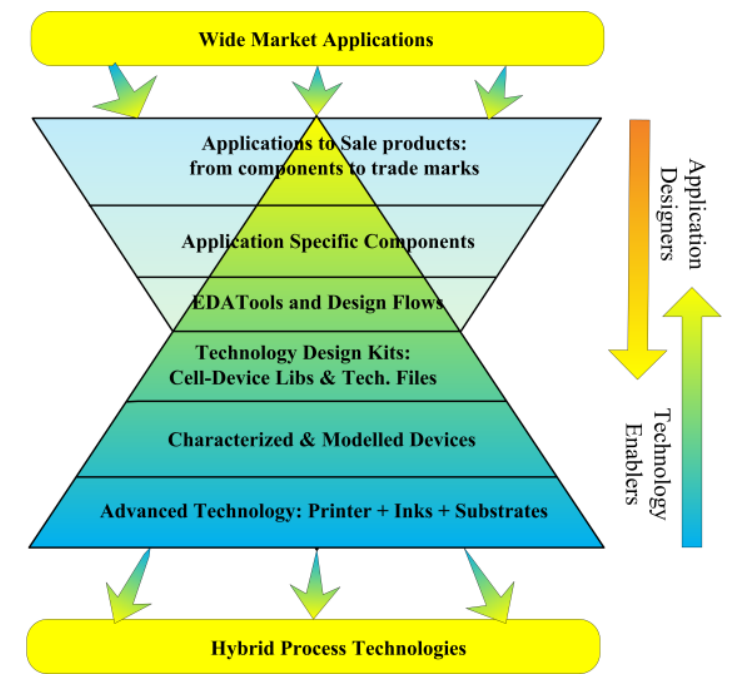

Fig. 12. Future view of the OTFT based large area electronics industry with a wide set of products for different applications that profit from a wide range of hybrid technologies available for its implementation in an open horizontal business model through the integration of their PDKs into the EDA tools.

Thirdly, trade-offs between performance and large area low cost processing need to be much better balanced. Strong opportunities can be opened for OTFTs with a suitable combination of mass-scale processes and suitable performances. Clearly, these two goals require trade-offs, since large-area manufacturing poses constraints for example on critical features, thus ultimately limiting performances. A clear challenge therefore is to develop methodologies characterized by a high patterning resolution, down to the micron size, while maintaining scalability, compatibility with cheap plastic foils and a reasonable throughput. Aggressive downscaling of features size to a sub-micron level may not be required, as back on the envelop estimations return tens of $\mathrm{MHz}$ operation of OTFT with micron scale channels and mobility in the 1 to 5 
$\mathrm{cm}^{2} \mathrm{~V}^{-1} \mathrm{~s}^{-1}$ if capacitive parasitism is kept under control. Overall, the challenge in this respect can be pinned down to the control of the device footprint and capacitive parasitism, possibly benefiting from approaches previously adopted in established technologies, such as self-alignment, while maintaining a suitably high yield.

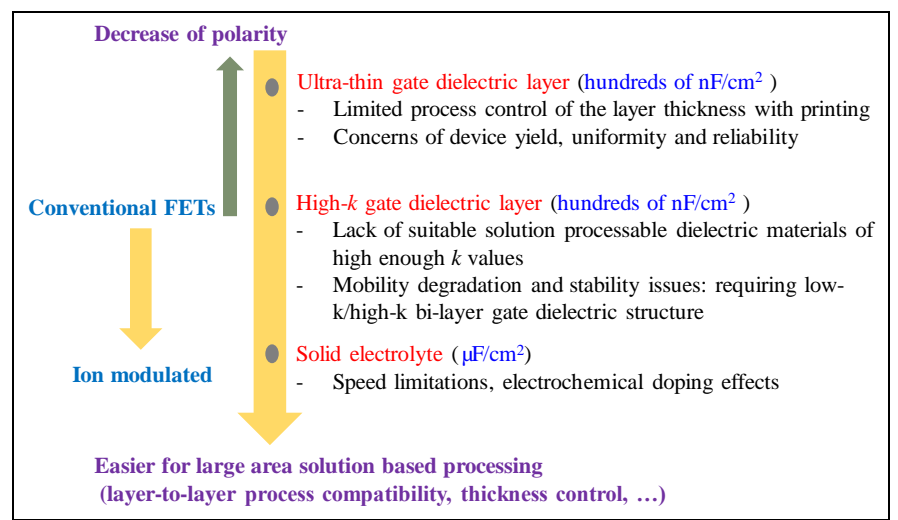

Fig. 13 Illustration of between reducing the operation voltage and having device structures compatible with large area solution based processing by using different gate dielectric materials.

Another trade-off is between reducing the operation voltage and having device structures compatible with large area solution based processing. Most of those envisioned applications of OTFTs are portable or wearable, requiring the devices to be integrated in severely power-constraint electronic systems with battery or a. c. field. This poses a stringent requirement on the operation voltage of the OTFT circuitry, which in principle should be scaled down to $3 \mathrm{~V}$, or even below. However, for OTFTs over large area substrates, especially based on solution processed multi-layer integration, the gate insulator layer needs to be thick enough (at least a few hundred nanometers) for the concern of device reliability and yield. Therefore, lots of work on using high $k$ gate dielectric material or even solid electrolytes to realize low voltage with a thick dielectric layer [81, 84]. The issue for high-k gate dielectric layer is that high-k dielectric materials could be unfavorable for carrier transport due to the broadening of the trap DOS at the semiconductor/dielectric interface by the formed dipole disorder [40]. The solid electrolyte can even induce electrochemical doping of the channel due to the presence of ions affect, causing reliability issues and slow operation speed [85]. As summarized in Fig. 13, for the conventional way of enlarging the gate dielectric capacitance to reduce the operation voltage, there is trade-off between processability and performance requirements. A promising solution is to reduce the sub-gap DOS at the channel through solution processed organic semiconductors, and thus very small gate dielectric capacitance can be used to realize low operation voltage [77, 112]. This approach provides the possibility of using a wide range of low- $k$ polymer dielectric materials in a relatively thick layer to achieve stable low voltage OTFTs $[69,74,75,88]$.

Last but not the least, focused applications needs to be clearly defined. The technology platform in FlexEnable (previously Plastic Logic) has demonstrated that there are no fundamental problems with the OTFT technology for commercialization. It is also shown that OTFT has huge market potential, and can completely change our view of where and how electronics is used in our daily lives. However, confidence in the OTFT technology might be increased if a greater number of fab or pilot lines existed with a predictable yield and uniformity. On the other hand, however, investments would not be put on constructing new fab or pilot lines or even upgrading existing fabs for OTFT manufacturing if no clear applications were seen. Therefore, with a near-overwhelming list of applications (both existing and new markets), OTFT technology companies need therefore to focus on the right applications. Building partnerships with other parts of the supply chain is critical to building an ecosystem for the OTFT technology, and allows technology companies to focus on their respective areas of expertise.

\section{CONCLUSIONS}

The review of research and development progresses in all aspects ranging from fundamental material and device research to manufacturing trials in last decades shows that solid basis has been formed for the OTFT technology to become commercially successful. However, to eventually become a widely adopted technology for the envisioned applications, there are still lots of challenges ahead to be addressed. The first one is to develop OTFT material stacks capable of producing high performance, stable and uniform OTFTs in large area compatible processes. The second challenge would be how to define standard material stacks, device /integration architectures, and manufacturing processes to allow the whole community to work on the common issues, and also reduce the entry barrier for the design community. How to balance the device performance and the large low cost manufacturability is also important to meet the application requirements. Finally, with a near-overwhelming list of applications (both existing and new markets), focused applications needs to be defined. To address these challenges, the whole community needs clear application and technology roadmaps and also standards in terms of materials, device/integration structures and processes to work together.

\section{ACKNOWLEDGMENT}

The authors would like to thank Dr. Ta-Ya Chu from National Research Council, Canada, and Dr. Jie Zhang from Changzhou Institute of Printed Electronics Industry, China for fruitful discussions at the panel discussion at the 2016 Computer Aided Design for Thin-Film Transistor Technologies (CAD-TFT) held in Beijing during October 26-28, 2016.

\section{REFERENCES}

[1].A. Nathan, A. Ahnood, J. Lai, and X. Guo, Large Area Electronics: Ed. J. Burghartz, John Wiley \& Sons, Ltd, 2013.

[2].R. A. Street, "Thin-film transistors," Adv. Mater., vol. 21, pp. 2007-2022, 2009.

[3].Y. Kuo, Thin Film Transistors: Amorphous Silicon Thin Film Transistor vol. 1: Springer Science \& Business Media, 2004. 
[4].S. D. Brotherton, Introduction to Thin Film Transistors: Physics and Technology of TFTs: Springer Science \& Business Media, 2013.

[5].K. Nomura, et al., "Room-temperature fabrication of transparent flexible thin-film transistors using amorphous oxide semiconductors," Nature, vol. 432, pp. 488-492, 2004.

[6]. T. Kamiya and H. Hosono, "Material characteristics and applications of transparent amorphous oxide semiconductors," NPG Asia Materials, vol. 2, pp. 15-22, 2010.

[7]. A. Nathan, et al., "Flexible electronics: the next ubiquitous platform," Proc. IEEE, vol. 100, pp. 1486-1517, 2012.

[8].L. Petti, et al., "Metal oxide semiconductor thin-film transistors for flexible electronics," Appl. Phys. Rev., vol.3, pp. 021303, 2016.

[9]. A. C. Arias, J. D. MacKenzie, I. McCulloch, J. Rivnay, and A. Salleo, "Materials and applications for large area electronics: solution-based approaches," Chem. Rev., vol 110, pp. 3-24, 2010.

[10]. H. T. Yi, M. M. Payne, J. E. Anthony, and V. Podzorov, "Ultra-flexible solution-processed organic field-effect transistors," Nat. Commun., vol. 3, pp. 1259, 2012.

[11]. K. Fukuda, et al., "Fully-printed high-performance organic thin-film transistors and circuitry on one-micron-thick polymer films," Nat. Commun., vol. 5, pp. 4147, 2014.

[12]. J. Y. Oh, et al., "Intrinsically stretchable and healable semiconducting polymer for organic transistors," Nature, vol. 539, pp. 411-415, 2016.

[13]. Y. Lee, M. Shin, K. Thiyagarajan, and U. Jeong, "Approaches to stretchable polymer active channels for deformable transistors," Macromolecules, vol. 49, pp. 433-444, 2015.

[14]. N. A. Minder, et al., "Tailoring the molecular structure to suppress extrinsic disorder in organic transistors," $A d v$. Mater., vol. 26, pp. 1254-1260, 2014.

[15].Z. B. Henson, K. Müllen, and G. C. Bazan, "Design strategies for organic semiconductors beyond the molecular formula," Nat. Chem., vol. 4, pp. 699-704, 2012.

[16]. J. Smith, et al., "Solution-processed organic transistors based on semiconducting blends," J. Mater. Chem., vol. 20, pp. 2562-2574, 2010.

[17]. T. Someya, A. Dodabalapur, J. Huang, K. C. See, and H. E. Katz, "Chemical and physical sensing by organic field - effect transistors and related devices," Adv. Mater., vol. 22, pp. 3799-3811, 2010.

[18]. Y. Guo, G. Yu, and Y. Liu, "Functional organic field-effect transistors," Adv. Mater., vol. 22, pp. 4427-4447, 2010.

[19]. P. Lin and F. Yan, "Organic thin-film transistors for chemical and biological sensing," Adv. Mater., vol. 24, pp. 34-51, 2012.

[20]. H. Sirringhaus, "Organic field-effect transistors: the path beyond amorphous silicon," Adv. Mater., vol. 26, pp. 1319-1335, 2014.
[21]. Y. Yuan, et al., "Ultra-high mobility transparent organic thin film transistors grown by an off-centre spin-coating method," Nat. Commun., vol. 5, pp. 3005, 2014.

[22]. B. H. Lee, G. C. Bazan, and A. J. Heeger, "Doping-induced carrier density modulation in polymer field - effect transistors," Adv. Mater., vol. 28, pp. 57-62, 2016.

[23]. K. Myny, et al., "An 8-bit, 40-instructions-per-second organic microprocessor on plastic foil," IEEE J. Solid-State Circuits, vol. 47, pp. 284-291, 2012.

[24]. W. Tang, et al., "Low-voltage $\mathrm{pH}$ sensor tag based on all solution processed organic field-effect transistor," IEEE Electron Device Lett., vol. 37, pp. 1002-1005, 2016.

[25]. W. Xiong, Y. Guo, U. Zschieschang, H. Klauk, and B. Murmann, "A 3-V, 6-bit C-2C digital-to-analog converter using complementary organic thin-film transistors on glass," IEEE J. Solid-State Circuits, vol. 45, pp. 1380-1388, 2010.

[26]. M. Kaltenbrunner, et al., "An ultra-lightweight design for imperceptible plastic electronics," Nature, vol. 499, pp. 458-463, 2013.

[27]. B. C.-K. Tee, et al., "A skin-inspired organic digital mechanoreceptor," Science, vol. 350, pp. 313-316, 2015.

[28]. R. Street, et al., "From printed transistors to printed smart systems," Proc. IEEE, vol. 103, pp. 607-618, 2015.

[29]. H. Klauk, "Organic thin-film transistors," Chem. Soc. Rev., vol. 39, pp. 2643-2666, 2010.

[30]. J. Zaumseil and H. Sirringhaus, "Electron and ambipolar transport in organic field-effect transistors," Chem. Rev., vol. 107, pp. 1296-1323, 2007.

[31]. Y. Wen, Y. Liu, Y. Guo, G. Yu, and W. Hu, "Experimental techniques for the fabrication and characterization of organic thin films for field-effect transistors," Chem. Rev., vol. 111, pp. 3358-3406, 2011.

[32].H. Lee, H. H. Choi, D. H. Kim, and K. Cho, "Microstructure dependent bias stability of organic transistors," Adv. Mater., vol. 26, pp. 1660-1680, 2014.

[33]. C. Reese, M. Roberts, M.-m. Ling, and Z. Bao, "Organic thin film transistors," Mater. Today, vol. 7, pp. 20-27, 2004.

[34]. G. Gelinck, P. Heremans, K. Nomoto, and T. D. Anthopoulos, "Organic transistors in optical displays and microelectronic applications," Adv. Mater., vol. 22, pp. 3778-3798, 2010.

[35]. A. Facchetti, "Semiconductors for organic transistors," Mater. Today, vol. 10, pp. 28-37, 2007.

[36]. K. J. Baeg, M. Caironi, and Y. Y. Noh, "Toward printed integrated circuits based on unipolar or ambipolar polymer semiconductors," Adv. Mater., vol. 25, pp. 4210-4244, 2013.

[37]. D. Natali and M. Caironi, "Charge Injection in solution-processed organic field - effect transistors: physics, models and characterization methods," $A d v$. Mater., vol. 24, pp. 1357-1387, 2012.

[38]. C. Liu, Y. Xu, and Y.-Y. Noh, "Contact engineering in organic field-effect transistors," Mater. Today, vol. 18, pp. 79-96, 2015.

[39].J.-F. Chang, et al., "Enhanced mobility of poly (3-hexylthiophene) transistors by spin-coating from 
high-boiling-point solvents," Chem. Mater., vol. 16, pp. 4772-4776, 2004.

[40]. J. Veres, S. D. Ogier, S. W. Leeming, D. C. Cupertino, and S. Mohialdin Khaffaf, "Low-k insulators as the choice of dielectrics in organic field - effect transistors," Adv. Funct. Mater., vol. 13, pp. 199-204, 2003.

[41]. B. S. Ong, Y. Wu, P. Liu, and S. Gardner, "High-performance semiconducting polythiophenes for organic thin-film transistors," J. Am. Chem. Soc., vol. 126, pp. 3378-3379, 2004.

[42]. I. McCulloch, et al., "Liquid-crystalline semiconducting polymers with high charge-carrier mobility," Nat. Mater., vol. 5, pp. 328-333, 2006.

[43]. Y.-Y. Noh, N. Zhao, M. Caironi, and H. Sirringhaus, "Downscaling of self-aligned, all-printed polymer thin-film transistors," Nat. Nano., vol. 2, pp. 784-789, 2007.

[44]. H. N. Tsao, et al., "Ultra-high mobility in polymer field-effect transistors by design," J. Am. Chem. Soc., vol. 133, pp. 2605-2612, 2011.

[45].X. Zhang, et al., "Molecular origin of high field-effect mobility in an indacenodithiophene-benzothiadiazole copolymer," Nat. Commun., vol. 4, pp. 2238, 2013.

[46]. D. Venkateshvaran, et al., "Approaching disorder-free transport in high-mobility conjugated polymers," Nature, vol. 515, pp. 384-388, 2014.

[47]. S. K. Park, T. N. Jackson, J. E. Anthony, and D. A. Mourey, "High mobility solution processed 6, 13-bis (triisopropyl-silylethynyl) pentacene organic thin film transistors," Appl. Phys. Lett., vol. 91, pp. 3514, 2007.

[48]. S. K. Park, D. A. Mourey, S. Subramanian, J. E. Anthony, and T. N. Jackson, "High-mobility spin-cast organic thin film transistors," Appl. Phys. Lett., vol. 93, pp. 043301, 2008.

[49]. H. Minemawari, et al., "Inkjet printing of single-crystal films," Nature, vol. 475, pp. 364-367, 2011.

[50]. T. Izawa, E. Miyazaki, and K. Takimiya, "Molecular ordering of high-performance soluble molecular semiconductors and re-evaluation of their field-effect transistor characteristics," Adv. Mater., vol. 20, pp. 3388-3392, 2008.

[51]. T. Sakanoue and H. Sirringhaus, "Band-like temperature dependence of mobility in a solution-processed organic semiconductor," Nat. Mater., vol. 9, pp. 736-740, 2010.

[52]. J. Smith, et al., "Solution-processed small molecule-polymer blend organic thin - film transistors with hole mobility greater than $5 \mathrm{~cm}^{2} / \mathrm{Vs}$," Adv. Mater., vol. 24, pp. 2441-2446, 2012.

[53]. K. L. McCall, et al., "High performance organic transistors using small molecule semiconductors and high permittivity semiconducting polymers," Adv. Funct. Mater., vol. 24, pp. 3067-3074, 2014.

[54]. E. G. Bittle, J. I. Basham, T. N. Jackson, O. D. Jurchescu, and D. J. Gundlach, "Mobility overestimation due to gated contacts in organic field-effect transistors," Nat. Commun., vol. 7, pp. 10908, 2016.

[55].X. Gao, et al., "Core-expanded naphthalene diimides fused with 2-(1,3-dithiol-2-ylidene) malonitrile groups for high-performance, ambient-stable, solution-processed n-channel organic thin film transistors," J. Am. Chem. Soc., vol. 132, pp. 3697-3699, 2010.

[56]. Y. Wen and Y. Liu, "Recent progress in n-channel organic thin-film transistors," Adv. Mater., vol. 22, pp. 1331-1345, 2010.

[57]. J. E. Anthony, A. Facchetti, M. Heeney, S. R. Marder, and X. Zhan, "n-type organic semiconductors in organic electronics," Adv. Mater., vol. 22, pp. 3876-3892, 2010.

[58]. R. Haddon, et al., "C60 thin film transistors," Appl. Phys. Lett., vol. 67, pp. 121-123, 1995.

[59]. R. Haddon, "C70 thin film transistors," J. Am. Chem. Soc., vol. 118, pp. 3041-3042, 1996.

[60].H. Yan, et al., "A high-mobility electron-transporting polymer for printed transistors," Nature, vol. 457, pp. 679-686, 2009.

[61].F. Zhang, et al., "Ultrathin film organic transistors: Precise control of semiconductor thickness via spin coating," Adv. Mater., vol. 25, pp. 1401-1407, 2013.

[62]. S. G. Bucella, et al., "Macroscopic and high-throughput printing of aligned nanostructured polymer semiconductors for $\mathrm{MHz}$ large-area electronics," Nat. Commun., vol. 6, pp. 8394, 2015.

[63].X. Guo, R. Sporea, J. Shannon, and S. Silva, "Down-scaling of thin-film transistors: opportunities and design challenges," ECS Trans., vol. 22, pp. 227-238, 2009.

[64]. J. Veres, S. Ogier, G. Lloyd, and D. de Leeuw, "Gate insulators in organic field-effect transistors," Adv. Mater., vol. 16, pp. 4543-4555, 2004.

[65]. A. Facchetti, M. H. Yoon, and T. J. Marks, "Gate dielectrics for organic field-effect transistors: new opportunities for organic electronics," Adv. Mater., vol. 17, pp. 1705-1725, 2005.

[66]. L. Feng, J. Anguita, W. Tang, J. Zhao, X. Guo, and S. R. P. Silva, "Room temperature grown high-quality polymer-like carbon gate dielectric for organic thin-film transistors", Adv. Electron. Mater., vol. 2, pp. 1500374, 2016.

[67]. F. Werkmeister and B. Nickel, "Towards flexible organic thin film transistors (OTFTs) for biosensing," J. Mater. Chem. B, vol. 1, pp. 3830-3835, 2013.

[68]. W. Huang, H. Fan, X. Zhuang, and J. Yu, "Effect of UV/ozone treatment on polystyrene dielectric and its application on organic field-effect transistors," Nanoscale Res. Lett., vol. 9, pp. 1-8, 2014.

[69]. L.-L. Chua, et al., "General observation of n-type field-effect behaviour in organic semiconductors," Nature, vol. 434, pp. 194-199, 2005.

[70]. S. Pyo, H. Son, K.-Y. Choi, M. H. Yi, and S. K. Hong, "Low-temperature processable inherently photosensitive polyimide as a gate insulator for organic thin-film transistors," Appl. Phys. Lett., vol. 86, pp. 133508, 2005.

[71]. L. Feng, et al., "Unencapsulated air-stable organic field effect transistor by all solution processes for low power vapor sensing," Sci. Rep., vol. 6, pp. 20671, 2016.

[72]. W. Tang, L. Feng, P. Yu, J. Zhao, and X. Guo, "Highly efficient all-solution-processed low-voltage organic transistor with a micrometer-thick low-k polymer gate 
dielectric layer," Adv. Electron. Mater., vol.5, pp. 1500454, 2016.

[73]. H. Sirringhaus, et al., "High-resolution inkjet printing of all-polymer transistor circuits," Science, vol. 290, pp. 2123-2126, 2000.

[74]. L. Feng, W. Tang, X. Xu, Q. Cui, and X. Guo, "Ultralow-voltage solution-processed organic transistors with small gate dielectric capacitance," IEEE Electron Device Lett., vol. 34, pp. 129-131, 2013.

[75]. R. Parashkov, et al., "All-organic thin-film transistors made of poly (3-butylthiophene) semiconducting and various polymeric insulating layers," J. Appl. Phys., vol. 95, pp. 1594-1596, 2004.

[76]. K. J. Baeg, et al., "Remarkable enhancement of hole transport in top-gated n-type polymer field-effect transistors by a high-k dielectric for ambipolar electronic circuits," Adv. Mater., vol. 24, pp. 5433-5439, 2012.

[77]. D. Khim, et al., "Facile route to control the ambipolar transport in semiconducting polymers," Chem. Mater., vol. 28, pp. 2287-2294, 2016.

[78]. J. Li, Z. Sun, and F. Yan, "Solution processable low-voltage organic thin film transistors with high- $\mathrm{k}$ relaxor ferroelectric polymer as gate insulator," $A d v$. Mater., vol. 24, pp. 88-93, 2012.

[79]. W. Tang, et al., "High-performance solution-processed low-voltage polymer thin-film transistors with low-/high-bilayer gate dielectric," IEEE Electron Device Lett., vol. 36, pp. 950-952, 2015.

[80]. K.-J. Baeg, et al., "Controlled charge transport by polymer blend dielectrics in top-gate organic field-effect transistors for low-voltage-operating complementary circuits," ACS Appl. Mater. Inter., vol. 4, pp. 6176-6184, 2012.

[81]. L. Herlogsson, et al., "Downscaling of organic field-effect transistors with a polyelectrolyte gate insulator," Adv. Mater., vol. 20, pp. 4708-4713, 2008.

[82]. J. H. Cho, et al., "Printable ion-gel gate dielectrics for low-voltage polymer thin-film transistors on plastic," Nat. Mater., vol. 7, pp. 900-906, 2008.

[83]. S. Li, W. Tang, W. Zhang, X. Guo, and Q. Zhang, "Cross-linked polymer-blend gate dielectrics through thermal click chemistry," Chem. Eur. J., vol. 21, pp. 17762-17768, 2015.

[84]. C. Wang, et al., "Thiol-ene cross-linked polymer gate dielectrics for low-voltage organic thin-film transistors," Chem. Mater., vol. 25, pp. 4806-4812, 2013.

[85]. X. Cheng, et al., "Air stable cross-linked cytop ultrathin gate dielectric for high yield low-voltage top-gate organic field-effect transistors," Chem. Mater., vol. 22, pp. 1559-1566, 2010.

[86]. S. Li, L. Feng, X. Guo, and Q. Zhang, "Application of thermal azide-alkyne cycloaddition (TAAC) reaction as a low temperature cross-linking method in polymer gate dielectrics for organic field-effect transistors," J. Mater. Chem. C, vol. 2, pp. 3517-3520, 2014.

[87]. S. Li, L. Feng, J. Zhao, X. Guo, and Q. Zhang, "Low temperature cross-linked, high performance polymer gate dielectrics for solution-processed organic field-effect transistors," J. Polym. Sci. Pol. Chem., vol. 6, pp. 5884-5890, 2015.

[88]. D. Boudinet, et al., "Influence of substrate surface chemistry on the performance of top-gate organic thin-film transistors, " J. Am. Chem. Soc., vol. 133, pp. 9968-9971, 2011.

[89].S. H. Han, et al., "Lifetime of organic thin-film transistors with organic passivation layers," Appl. Phys. Lett., vol. 88, pp. 073519, 2006.

[90]. M. Hong, et al., "Recent progress in large sized \& high performance organic TFT array," in SID Symposium Digest of Technical Papers, 2005, pp. 23-25.

[91].D. X. Long, et al., "Solution processed vanadium pentoxide as charge injection layer in polymer field-effect transistor with Mo electrodes," Org. Electron., vol. 17, pp. 66-76, 2015.

[92]. C.-A. Di, et al., "High-performance low-cost organic field-effect transistors with chemically modified bottom electrodes," J. Am. Chem. Soc., vol. 128, pp. 16418-16419, 2006.

[93]. Y. Xu, et al., "Planar-processed polymer transistors," Adv. Mater., vol. 28, pp. 8531-8537, 2016.

[94]. D. Gundlach, et al., "Contact-induced crystallinity for high-performance soluble acene-based transistors and circuits," Nat. Mater., vol. 7, pp. 216-221, 2008.

[95]. S. Burns, et al., "A scalable manufacturing process for flexible active-matrix e-paper displays," J. Soc. Inf. Display, vol. 13, pp. 583-586, 2005.

[96]. M. Mizukami, et al., "Flexible AMOLED panel driven by bottom-contact OTFTs," IEEE Electron Device Lett., vol. 27, pp. 249-251, 2006.

[97]. D. Gundlach, et al., "An experimental study of contact effects in organic thin film transistors," J. Appl. Phys., vol. 100, pp. 024509, 2006.

[98]. J. M. Shannon, R. A. Sporea, S. Georgakopoulos, M. Shkunov, and S. R. P. Silva, "Low-field behavior of source-gated transistors," IEEE Trans. Electron Devices, vol. 60, pp. 2444-2449, 2013.

[99]. R. Sporea, M. Trainor, N. Young, J. Shannon, and S. Silva, "Source-gated transistors for order-of-magnitude performance improvements in thin-film digital circuits," Sci. Rep., vol. 4, pp. 4295, 2014.

[100].X. Guo and J. Shannon, "Current-mode logic in organic semiconductor based on source-gated transistors," IEEE Electron Device Lett., vol. 30, pp. 365-367, 2009.

[101].W. Tang, et al., "Top-gate dry-etching patterned polymer thin-film transistors with a protective layer on top of the channel," IEEE Electron Device Lett., vol. 36, pp. 59-61, 2015.

[102].C. B. Park, et al., "Commercially applicable, solution-processed organic TFT and its backplane application in electrophoretic displays," Solid-State Electron., vol. 111, pp. 227-233, 2015.

[103]. S. Steudel, K. Myny, S. De Vusser, J. Genoe, and P. Heremans, "Patterning of organic thin film transistors by oxygen plasma etch," Appl. Phys. Lett., vol. 89, p. $183503,2006$.

[104].M. Noda, et al., "An OTFT-driven rollable OLED display," J. Soc. Inf. Display, vol. 19, pp. 316-322, 2011. 
[105].http://www.plasticlogic.com/products/

[106].S. J. A. Soua, et al., "Programmable logic circuits for functional integrated smart plastic systems," Org. Electron., vol. 15, p. 9, 2014.

[107]. T. N. Ng, et al., "Printed dose-recording tag based on organic complementary circuits and ferroelectric nonvolatile memories," Sci. Rep., vol. 5, pp. 13457, 2015.

[108].T. N. Ng, et al., "Pulsed voltage multiplier based on printed organic devices," Flexible and Printed Electronics, vol. 1, pp. 015002, 2015.

[109]. S. Mutlu, I. Haydaroglu, and A. O. Sevim, "Realization of polymer charge pump circuits using polymer semiconductors," Org. Electron., vol. 12, pp. 312-321, 2011.

[110].H. I. M. Kunii, and J.-I. Hanna, "Solution-processed, low-voltage polycrystalline organic field-effect transistor fabricated using highly ordered liquid crystal with low-k gate dielectric," IEEE Electron Device Lett., vol. 37, pp. 486 - 488, 2016.

[111].L. Feng, C. Jiang, H. Ma, X. Guo, and A. Nathan, "All ink-jet printed low-voltage organic field-effect transistors on flexible substrate," Org. Electron., vol. 38, pp. 186-192, 2016.

[112].X. Guo, et al., "Fully Printable Organic Thin-Film Transistor Technology for Sensor Transducer," in Smart Sensors and Systems, ed: Springer, 2015, pp. 47-59.

[113].S. Preradovic, and N. Karmakar, "Chipless RFID tag with integrated sensor," Proc. IEEE Sensors, Nov. 2010, pp. 1277-1281.

[114].M. C. M. Oliverosa, et al., "Photosensitive chipless radio-frequency tag for low-cost monitoring of light-sensitive goods," Sens. Actuators B, Chem., vol. 223, pp. 839-845, 2016.

[115]. Y. Taur, and T. H. Ning, Fundamentals of Modern VLSI Devices: Cambridge University Press, 2013.

[116]. V. Wagner, P. Wöbkenberg, A. Hoppe, and J. Seekamp, "Megahertz operation of organic field-effect transistors based on poly (3-hexylthiopene)," Appl. Phys. Lett., vol. 89, pp. 243515, 2006.

[117].M. Caironi, Y. Noh, and H. Sirringhaus, "Frequency operation of low-voltage, solution-processed organic field-effect transistors," Semicond. Sci. Technol., vol. 26, pp. 034006, 2011.

[118].T. Zaki, et al., "S-parameter characterization of submicrometer low-voltage organic thin-film transistors," IEEE Electron Device Lett., vol. 34, pp. 520-522, 2013.

[119]. M. Kitamura and Y. Arakawa, "High current-gain cutoff frequencies above $10 \mathrm{MHz}$ in n-channel C60 and p-channel pentacene thin-film transistors," Jpn. J. Appl. Phys., vol. 50, pp. 01BC01, 2011.

[120].T. Uemura, et al., "Split-gate organic field-effect transistors for high-speed operation," Adv. Mater., vol. 26, pp. 2983-2988, 2014.

[121]. M. Uno, B. Cha, Y. Kanaoka, and J. Takeya, "High-speed organic transistors with three-dimensional organic channels and organic rectifiers based on them operating above $20 \mathrm{MHz}$," Org. Electron., vol. 20, pp. 119-124, 2015.

[122]. K. Nakayama, et al., "High-mobility organic transistors with wet-etch-patterned top electrodes: a novel patterning method for fine-pitch integration of organic devices," Adv. Mater. Interfaces, vol. 1, pp. 1300124, 2014.

[123].M. Uno, et al., "High-speed organic single-crystal transistors gated with short-channel air gaps: Efficient hole and electron injection in organic semiconductor crystals," Org. Electron., vol. 14, pp. 1656-1662, 2013.

[124]. A. Perinot, et al., "Direct-written polymer field-effect transistors operating at $20 \mathrm{MHz}, "$ Sci. Rep., vol. 6, pp. 38941, 2016.

[125].S. Higgins, et al., "Self-aligned organic field-effect transistors on plastic with picofarad overlap capacitances and megahertz operating frequencies," Appl. Phys. Lett., vol. 108, pp. 023302, 2016.

[126].H. Kang, R. Kitsomboonloha, J. Jang, and V. Subramanian, "High-performance printed transistors realized using femtoliter gravure - printed sub-10 $\mu \mathrm{m}$ metallic nanoparticle patterns and highly uniform polymer dielectric and semiconductor layers," $A d v$. Mater., vol. 24, pp. 3065-3069, 2012.

[127].S. P. Senanayak, et al., "Self-assembled nanodielectrics for high-speed, low-voltage solution-processed polymer logic circuits," Adv. Electron. Mater., vol. 1, pp. 1500226, 2015.

[128].C.-H. Kim, Y. Bonnassieux, and G. Horowitz, "Compact DC modeling of organic field-effect transistors: Review and perspectives," IEEE Trans. Electron Devices, vol. 61, pp. 278-287, 2014.

[129].P. Stallinga, "Electronic transport in organic materials: comparison of band theory with percolation/(variable range) hopping theory," Adv. Mater., vol. 23, pp. 3356-3362, 2011.

[130]. O. Marinov, M. J. Deen, U. Zschieschang, and H. Klauk, "Organic thin-film transistors: Part I-Compact DC modeling," IEEE Trans. Electron Devices, vol. 56, pp. 2952-2961, 2009.

[131].L. Li, H. Marien, J. Genoe, M. Steyaert, and P. Heremans, "Compact model for organic thin-film transistor," IEEE Electron Device Lett., vol. 31, pp. 210-212, 2010.

[132].S. Sambandan, et al., "Compact model for forward subthreshold characteristics in polymer semiconductor transistors," J. Appl. Phys., vol. 106, pp. 084501, 2009.

[133].L. Colalongo, "SQM-OTFT: A compact model of organic thin-film transistors based on the symmetric quadrature of the accumulation charge considering both deep and tail states," Org. Electron., vol. 32, pp. 70-77, 2016.

[134].H. Klauk, et al., "Contact resistance in organic thin film transistors," Solid State Electron., vol. 47, pp. 297-301, 2003.

[135].C. H. Kim, et al., "A compact model for organic field-effect transistors with improved output asymptotic behaviors," IEEE Trans. Electron Devices, vol. 60, pp. 1136-1141, 2013. 
[136].M. Shur, "The compact models and parameter extraction for thin film transistors," ECS Trans., vol. 75, pp. 171-178, 2016.

[137]. X. Cheng, S. Lee, G. Yao, and A. Nathan, "TFT compact modelling," IEEE/OSA J. Disp. Technol., vol. 12, pp. 898 - 906, 2016.

[138].SmartSPICE Users Manual. Santa Clara, CA,: Silvaco Int., 2010.

[139].D. Bode, et al., "Noise-margin analysis for organic thin-film complementary technology," IEEE Trans. Electron Devices, vol. 57, pp. 201-208, 2010.

[140]. T.-C. Huang, et al., "Pseudo-CMOS: A design style for low-cost and robust flexible electronics," IEEE Trans. Electron Devices, vol. 58, pp. 141-150, 2011.

[141].I. Nausieda, et al., "Dual threshold voltage organic thin-film transistor technology," IEEE Trans. Electron Devices, vol. 57, pp. 3027-3032, 2010.

[142].H. Marien, M. Steyaert, E. Veenendaal, and P. Heremans, "On the other applications of organic electronics on foil," IEEE Solid-State Circuits Magazine, vol. 4, pp. 43-49, 2012.

[143].J. Zhao, Q. Cui, and X. Guo, "An analytical yield model for zero-load thin-film transistor logic circuits," IEEE Electron Device Lett., vol. 35, pp. 1269-1271, 2014.

[144].Q. Zhao, et al., "Noise margin modeling for zero-load TFT circuits and yield estimation," IEEE Trans. Electron Devices, vol. 63, pp. 684-690, 2016.

[145].http://projects.si2.org/opdkc_index.php

[146].M. Llamas, et al., "Top-down design flow for application specific printed electronics circuits (ASPECs)," in Design of Circuits and Integrated Circuits (DCIS), 2014 Conference on, 2014, pp. 1-5.

[147].J. Noh, et al., "Key issues with printed flexible thin film transistors and their application in disposable RF sensors," Proc. IEEE, vol. 103, pp. 554-566, 2015.

[148]. V. Fiore, et al., "An integrated 13.56-MHz RFID tag in a printed organic complementary TFT technology on flexible substrate," IEEE Transactions on Circuits and Systems I: Regular Papers, vol. 62, pp. 1668-1677, 2015.

[149]. M. Mashayekhi, et al., "Development of a standard cell library and ASPEC design flow for Organic Thin Film Transistor technology," in Design of Circuits and Integrated Circuits (DCIS), 2014 Conference on, 2014, pp. 1-6.

[150].S. G. Uppili, D. R. Allee, S. M. Venugopal, L. T. Clark, and R. Shringarpure, "Standard cell library and automated design flow for circuits on flexible substrates," in 2009 Flexible Electronics \& Displays Conference and Exhibition, 2009, pp. 1-5.

[151]. K. Ishida, et al., "User customizable logic paper (UCLP) with sea-of transmission-gates (SOTG) of 2-V organic CMOS and ink-jet printed interconnects," IEEE J. Solid-State Circuits, vol. 46, pp. 285-292, 2011.

[152].A. Sou, et al., "Programmable logic circuits for functional integrated smart plastic systems," Org. Electron., vol. 15, pp. 3111-3119, 2014.

[153].K. Myny, et al., "8b thin-film microprocessor using a hybrid oxide-organic complementary technology with inkjet-printed P2ROM memory," in 2014 IEEE
International Solid-State Circuits Conference Digest of Technical Papers (ISSCC), 2014, pp. 486-487.

[154].J. Carrabina, M. Mashayekhi, J. Pallares, and L. Teres, "Inkjet-configurable gate arrays (IGA)," IEEE Trans. Emerg. Topics Comput., vol. PP, 2016.

[155].Z. T. Zhu, et al., "A simple poly (3,4-ethylene dioxythiophene)/poly (styrene sulfonic acid) transistor for glucose sensing at neutral pH," Chem. Commun., vol. 13, pp. 1556-1557, 2004.

[156].P. Lin, X. Luo, I. Hsing, and F. Yan, "Organic electrochemical transistors integrated in flexible microfluidic systems and used for label-free DNA sensing," Adv. Mater., vol. 23, pp. 4035-4040, 2011.

[157].P. Lin, F. Yan, J. Yu, H. L. Chan, and M. Yang, "The application of organic electrochemical transistors in cell-based biosensors," Adv. Mater., vol. 22, pp. 3655-3660, 2010.

[158].D. Khodagholy, et al., "In vivo recordings of brain activity using organic transistors," Nat. Commun., vol. 4, pp. $1575,2013$.

[159].R.-X. He, et al., "Detection of bacteria with organic electrochemical transistors," J. Mater. Chem., vol. 22, pp. 22072-22076, 2012.

[160].M. Y. Mulla, et al., "Capacitance-modulated transistor detects odorant binding protein chiral interactions," Nat. Commun., vol. 6, pp. 6010, 2015.

[161].S. Wild, G. Roglic, A. Green, R. Sicree, and H. King, "Global prevalence of diabetes estimates for the year 2000 and projections for 2030," Diabetes Care, vol. 27, pp. 1047-1053, 2004.

[162].C. Liao, C. Mak, M. Zhang, H. L. Chan, and F. Yan, "Flexible organic electrochemical transistors for highly selective enzyme biosensors and used for saliva testing," Adv. Mater., vol. 27, pp. 676-681, 2015.

[163].H. Sirringhaus, "Reliability of organic field-effect transistors," Adv. Mater., vol. 21, pp. 3859-3873, 2009.

[164]. M. Nikolka, et al., "High operational and environmental stability of high-mobility conjugated polymer field-effect transistors achieved through the use of molecular additives," Nat. Mater., in press. DOI:10.1038/nmat4785.

[165].K. Fukuda, et al., "Printed organic transistors with uniform electrical performance and their application to amplifiers in biosensors," Adv. Electron. Mater., vol. 1, pp. 1400052, 2015.

[166].M. Guerin, et al., "High-gain fully printed organic complementary circuits on flexible plastic foils," IEEE Trans. Electron Devices, vol. 58, pp. 3587-3593, 2011.

[167]. Y. Xu, C. Liu, D. Khim, and Y.-Y. Noh, "Development of high-performance printed organic field-effect transistors and integrated circuits," Phys. Chem. Chem. Phys., vol. 17, pp. 26553-26574, 2015.

[168].[TDK4PE] Technology Design Kit for Printed Electronics. EC project TDK4PE. Available: http://www.tdk4pe.eu/

[169].[FHE-PDK] Flexible Hybrid Electronics Process Design Kit by GeorgiaTech. Available: http://flex.gatech.edu/design-modeling-simulation 